\title{
FREE CROSSED RESOLUTIONS OF GROUPS AND PRESENTATIONS OF MODULES OF IDENTITIES AMONG RELATIONS
}

\author{
RONALD BROWN AND ABDUL RAZAK SALLEH
}

\begin{abstract}
The paper gives formulae for a module presentation of the module of identities among relations for a presentation of a group, in terms of information on 0- and 1-combings of the Cayley graph. These formulae are seen as a special case of formulae for extending a partial free crossed resolution of a group, given a partial contracting homotopy of the universal cover of the partial resolution.
\end{abstract}

\section{Introduction}

The initial motivation for this work was to determine, in an algorithmic mode, generators and relations for the $G$-module $\pi(\mathcal{P})$ of identities among relations for a presentation $\mathcal{P}=$ $\langle X \mid \omega: R \rightarrow F(X)\rangle$ of a group $G$. Here we regard $R$ as a set disjoint from $F(X)$, and $\omega$ gives the corresponding element of $F(X)$. Recall that $\pi(\mathcal{P})$ is given algebraically as the kernel of $\delta_{2}: C(R) \rightarrow F(X)$, the free crossed module of the presentation, and is given geometrically as $\pi_{2}(K(\mathcal{P}))$, the second homotopy group of the cell complex of the presentation [17].

Our main results imply a formula as follows.

Theorem 1.1. The module $\pi(\mathcal{P})$ is generated as $G$-module by elements

$$
\delta_{3}[g, r]=\left(k_{1}(g, \omega r)\right)^{-1} r^{(\sigma g)^{-1}}
$$

for all $g \in G, r \in R$, where

(i) $\sigma: G \rightarrow F(X)$ is a section of the quotient mapping $\varphi: F(X) \rightarrow G$,

(ii) $k_{1}$ is a morphism $F(\tilde{X}) \rightarrow C(R)$ from the free groupoid on $\widetilde{X}$, the Cayley graph of the presentation, to the free crossed module of the presentation, such that $\delta_{2} k_{1}(g, x)=$ $(\sigma g) x(\sigma(g(\varphi x)))^{-1}$, for all $x \in X, g \in G$.

The identities $\delta_{3}[g, r]$ may be seen as separation elements in the geometry of the Cayley graph with relators, as defined in Sections 2 and 4. It is easy to see from properties (i), (ii) and the first crossed module rule that these elements all lie in $\pi(\mathcal{P})$. Thus the main feature of the theorem is that these elements generate this module. We shall also show that if the section $\sigma$ is determined by a tree in the Cayley graph, then these elements generate $\pi(\mathcal{P})$ additively.

Part of this work was supported by EPSRC Grant GR/J63552 'Non abelian homological algebra',1994-6, awarded to R. Brown and T. Porter.

The second author thanks the Association of Commonwealth Universities for support as a Commonwealth Fellow in 1994-95, the School of Mathematics, University of Wales, Bangor, for hospitality in this period, and Universiti Kebangsaan Malaysia, for granting the sabbatical leave.

Received 7 January 1999, revised 16 February 1999; published 23 April 1999.

1991 Mathematics Subject Classification 20F05, 20J05, 20L10, 18G50, 57M07

(C) 1999, Ronald Brown and Abdul Razak Salleh 
The identities $\delta_{3}[g, r]$ will be seen to arise from a boundary mapping $\delta_{3}: C_{3}(I) \rightarrow C(R)$ from the free $G$-module on a set $I$ bijective with $G \times R$, with basis elements written $[g, r], g \in G, r \in R$. The set $\delta_{3}(I)$ is usually not a minimal set of generators (many of them may even be trivial). So we suppose given a subset $J$ of $I$, determining a free $G$-module $C_{3}(J)$, with the property that $\delta_{3}(J)$ also generates $\pi(\mathcal{P})$, and then seek relations among these generators $\delta_{3}(J)$.

Theorem 1.2. A $G$-module generating set of relations among these generators $\delta_{3}(J)$ of $\pi(\mathcal{P})$ is given by

$$
\delta_{4}[g, \gamma]=-k_{2}\left(g, \delta_{3} \gamma\right)+\gamma \cdot g^{-1}
$$

for all $g \in G, \gamma \in J$, where $k_{2}: C(\widetilde{R}) \rightarrow C_{3}(J)$ is a morphism from the free crossed $F(\tilde{X})$-module on $\widetilde{\delta}_{2}: G \times R \rightarrow F(\widetilde{X})$ such that $k_{2}$ kills the operation of $F(\widetilde{X})$ and is determined by a choice of writing the generators $\delta_{3}[g, r] \in \delta_{3}(I)$ for $\pi(\mathcal{P})$ in terms of the elements of $\delta_{3}(J)$.

It will be noted that both these results use the language of groupoids. Morphisms from a groupoid to a group can be a convenient way of combining graphical and group theoretic information.

In Section 2 we shall explain the terms in these theorems in enough detail for the reader to follow an explicit calculation for the standard presentation of the group $S_{3}$ in Section 3 . We give this example because it is sufficiently complex to illustrate important features of the calculations, and sufficiently simple that the calculations can be carried out by hand.

In the example of $S_{3}$ Theorem 1.1 gives eighteen generators for the module $\pi(\mathcal{P})$; we show this number can be reduced to four ${ }^{1}$. This set of generators was already known. The rewriting involved in this reduction process and Theorem 1.2 are then used to construct the next level of syzygies. This initially yields twenty-four relations among identities, which are then shown to be reducible to five. We are not aware of any previous determination of relations among generators for this module of identities. These calculations have been extended by hand, but with different choices, by two further stages in [26]. The numbers of generators in the next dimensions are six and then seven.

The reader will notice the analogy between the formulae in these theorems - they are in fact special cases of Corollary 10.3, which computes higher-order syzygies inductively. The context of that result is that of free crossed resolutions, universal covers of crossed resolutions, and contracting homotopies of such universal covers. Once this machinery has been set up, the result becomes almost tautologous. It states that the pair consisting of a partial free crossed resolution and a partial contracting homotopy of its universal cover can be extended by one step, and hence indefinitely.

A sequel to this paper by Heyworth and Wensley [28] will show how the part of the procedure required for Theorem 1.1 can be implemented as a 'logged Knuth-Bendix procedure', which terminates when the monoid rewrite system associated with the presentation completes. This step has applications for infinite groups, and the resulting infinite set of generators for the module of identities may in some cases be reducible by hand.

A further paper by Heyworth and Reinert [27] will show how the reduction process required for Theorem 1.2 can be implemented in the case of finite groups by using generalised

${ }^{1}$ Note added in proof: In fact, G. J. Ellis and I. Kholodna have recently shown that this number may be reduced to 3 . 
Gröbner basis procedures for integral group rings. This allows a wide range of computations, for example for standard presentations of the groups $Q_{8}, A_{5}, B(2,3)$. These further papers will also discuss implementation issues.

The partial contracting homotopies are given by functions $h_{i}$ for $i<n$ with appropriate properties. In fact $h_{0}$ corresponds to a 0 -combing, and $h_{1}$ is analogous to a 1-combing; from these we obtain the functions $\sigma, k_{1}$ of the theorems. The algebra of such functions is shown to be nicely handled in the context of the free groupoid $F(\widetilde{X})$ on the Cayley graph and the free crossed module $\tilde{\delta}_{2}: C(\widetilde{R}) \rightarrow F(\widetilde{X})$. We show that this crossed module is the fundamental crossed module of the universal cover of the geometric 2-complex of the presentation. The groupoid approach is required to utilise the vertices of the universal cover.

In terms of chain complexes of modules rather than crossed complexes, a computation of the module of identities among relations for the presentation $\langle X \mid R\rangle$ of the group $G$ may be seen as that of computing an extension of the partial resolution of $\mathbb{Z}$

$$
(\mathbb{Z} G)^{R} \rightarrow(\mathbb{Z} G)^{X} \rightarrow \mathbb{Z} G \rightarrow \mathbb{Z}
$$

where the first morphism is given by the Whitehead-Fox derivative $(\partial r / \partial x)$ [40, Lemma 8]; [23]. The process of extending a partial resolution is more difficult than that of just giving a resolution. There is in fact considerable work on constructing resolutions of groups, some of it for 2-groups mod 2 [21], and other results using homological perturbation theory, particularly by Larry Lambe and colleagues [35]. It is not clear how these methods apply to the problem of extending partial resolutions. Work of Groves [24] constructs a resolution from a complete rewrite system for a monoid presentation of the group, rather than directly from a group presentation. However, as mentioned above, complete rewrite systems are relevant to the computation of $k_{1}$ of Theorem 1.1 .

Gruenberg in [25] constructs a free $\mathbb{Z} G$-module resolution dependent functorially for fixed $G$ on the exact sequence $1 \rightarrow N \rightarrow F \rightarrow G \rightarrow 1$. If $F$ is free on $X$ and $Y$ is a set of free generators of the group $N$, then the number of generators in dimension $n$ is $|Y|^{n-1}$ or $|Y|^{n-1}|X|$ according to whether $n$ is even or odd. Thus the Gruenberg resolution is not isomorphic to the module resolution obtained from our crossed resolution by the process of $[40,15]$ (part of which is used here in Theorem 2.5). Gruenberg's work was pointed out to us by Justin Smith, who has further work on the resolution in [38].

Our method yields a resolution that is dependent only on the presentation and two initial sets of choices. More generally, we can obtain a free crossed resolution that is dependent only on the first $n$ stages of a free crossed resolution and a contracting homotopy up to that stage of the universal cover.

In the final section we show how these methods give rise to the standard crossed resolution of a group $G$, and to a small crossed resolution of a finite cyclic group. The resolution is in each case found through the construction of the universal cover and its contracting homotopy.

It is interesting to compare our methods with the methods of pictures for calculating the generators of $\pi(\mathscr{P})$ (see for example $[\mathbf{1}, \mathbf{1 7}, \mathbf{3 1}, \mathbf{3 6}]$ ). These methods use the geometry of the relations nicely; they have been very successful in this field, and can prove more efficient than ours for finding generators of $\pi(\mathcal{P})$. However, they seem more difficult to carry out in higher dimensions, for the following reasons.

The picture methods use 2-dimensional rewrite rules described by 'pictures' to reduce spherical elements to a combination of standard elements. The full information on the way in which these rewrites are used in a particular example is essentially 3-dimensional, and it can thus be difficult to visualise or to record combinations of such rewrites, and their dependencies. For our purposes this rewriting information must be recorded completely 
(see Tables 2, 4 of Section 3), since it is used to construct the next stage of a contracting homotopy; this use of the complete record is one reason for the apparently cumbersome nature of the calculations. Thus there are problems in extending the picture method to determine 3-syzygies, whereas our purely algebraic method is essentially uniform over dimensions, giving rise mainly to computational problems. This suggests that in dimension 2 , our methods should be seen as complementary to those of pictures.

The method of pictures has also been applied successfully to determine generators for the module of identities among relations for various constructions on groups (see, for example, [1]). In contrast, the only general construction on crossed resolutions which has so far been applied is the tensor product $[\mathbf{1 4}, \mathbf{1 8}, \mathbf{3 9}]$ - given free crossed resolutions $C, C^{\prime}$ of two groups $G, H$, the tensor product $C \otimes C^{\prime}$ gives a free crossed resolution of their product $G \times H$, and so a presentation of the module of identities for the standard combined presentation of the product. An application is given in [18].

There are three basic planks in our approach.

\section{(i) Crossed complexes}

Crossed complexes form an analogue of chain complexes, but with non-abelian features in dimensions 1 and 2. These features allow crossed complexes to combine many of the advantages of chain complexes with an ability to contain the information involved in a presentation of a group. So one can model many of the standard techniques of homological algebra, such as uniqueness up to homotopy of a free crossed resolution. Further, this technique may be combined with a non-abelian version of the traditional notion of 'chains of syzygies'; this version takes account of the facts that free groups are non-abelian, and that a normal subgroup $N$ of a group $F$ is in general non-abelian and admits an operation of $F$ on $N$ which is crucial in discussing presentations. Crossed complexes, unlike chain complexes, allow for 'free' models of this inclusion $N \rightarrow F$ (see [17]), and so give an intuitive algebraic model of chains of syzygies in this non-abelian case. An account of uses of crossed complexes up to 1981 is given in [13].

A small free crossed resolution is convenient for calculations of non-abelian extensions [18] and of the cohomology class of a crossed module [19, 20]. A free crossed resolution $C$ of $G$ determines a free $\mathbb{Z} G$ resolution $\Delta C$ of $\mathbb{Z}$ in the usual sense $[40,16]$. The crossed resolution $C$ with its free basis carries more information than $\Delta C$; for example, it includes a presentation of $G$.

\section{(ii) Algebraic models of the geometry of covering spaces}

Philip Higgins pointed out in 1964 [29] how presentations of groupoids could be applied to group theory. The geometric basis of the argument is that the theory of covering spaces is more conveniently handled if one uses groupoids rather than groups, since there is a purely algebraic notion of covering morphism of a groupoid which nicely models the geometry (see [4]). Covering morphisms of a group or groupoid $G$ are equivalent to operations of $G$ on sets.

In the same way, to apply crossed complexes to covering spaces we require crossed complexes of groupoids, not just of groups. Such general crossed complexes were also found essential in [12] for certain higher-order Van Kampen Theorems, so the basic definitions and applications are already known. This allows us to bring in techniques not only of presentations of groupoids, as discussed in [29], but also of free crossed resolutions determined by such a presentation. 
In effect, we are giving a suitable algebraic framework in which to place the geometry of the Cayley graph of a generating set of the group, but including the relations as well as the generators of the presentation, and indeed including higher-order syzygies, as these are constructed. This algebraic framework also conveniently models the geometry of the universal cover of a cell complex.

A crucial tool for our methods is the fact that a covering crossed complex of a free crossed complex is again a free crossed complex, on the 'covering generators' (Theorem 9.2). This models the geometry of CW-complexes. The result is crucial because it enables us to define morphisms and homotopies by their values on the free generators. Our proof relies on a result of Howie [32].

\section{(iii) Contracting homotopies}

The key point is that the previous techniques allow us to discuss free crossed resolutions of contractible groupoids, for example the universal covering groupoid of the original group. A free crossed resolution of a contractible groupoid will have contracting homotopies, and our method proceeds by the construction of such homotopies. This method is applied to truncated crossed complexes, and in particular to crossed modules. The usual slogan is to choose generators for the kernel and so kill homotopy groups; this fails to tell us how to choose these generators. Instead, we construct a crossed complex whose universal cover is a home for a contracting homotopy. This 'tautologously' yields generators of kernels. The method was suggested by lectures at Bangor of L.A. Lambe, on homological perturbation theory - in that context the construction of homotopies is crucial.

In order to make this method clear, we need the basics of the theory of presentations and of identities among relations for groupoids. We give the key features, largely without proofs, in Section 4. This leads to the theory of crossed modules of groupoids in Section 5.

The definition of crossed complexes is given in Section 6, and the definitions and results on covering morphisms that we need are presented in Sections 7-9. The notion of homotopy for crossed complexes is presented in Section 10, yielding results which justify the exposition of Section 2. The final section, 11, gives two more applications.

We would like to thank Anne Heyworth and Emma Moore for discussions on this material which led to the exposition in Sections 2 and 11, and Chris Wensley and a referee for helpful comments.

We finally remark that this paper should be seen as a part of the general programme on higher dimensional group theory described in [8].

\section{The computational procedure}

The purpose of this section is to state the computational procedure in as direct a way as we can. The theoretical underpinning is left to later sections. We hope that this will make it easier for the reader.

Let $\mathcal{P}=\langle X \mid \omega: R \rightarrow F(X)\rangle$ be a presentation of a group $G$. The advantages of using the function $\omega$ are (i) to allow for the possibility of repeated relations, and (ii) to distinguish between an element $r \in R$ and the corresponding element $\omega(r) \in F(X)$. We shall be concerned with the following diagram, in which $p_{0} \widetilde{\varphi}=\varphi p_{1}, p_{1} \widetilde{\delta}_{2}=\delta_{2} p_{2}, p_{2} \widetilde{\delta}_{3}=\delta_{3} p_{3}$. The parts of this diagram will be developed in paragraphs $2.1-2.12$ below. 


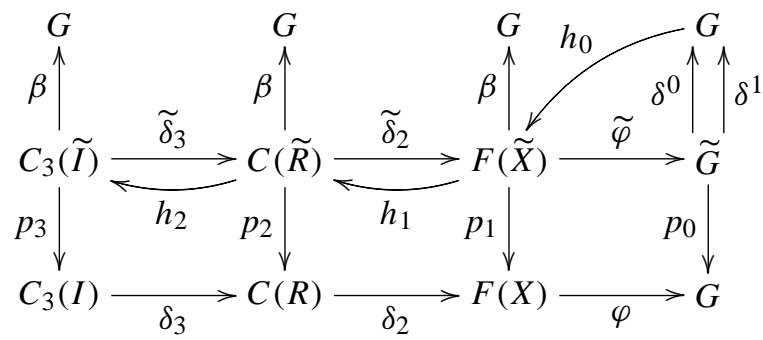

2.1. $\varphi: F(X) \rightarrow G$ is the canonical morphism from the free group on $X$ to $G$ given by the set of generators.

2.2. $\delta_{2}: C(R) \rightarrow F(X)$ is the free crossed $F(X)$-module on the function $\omega$.

Thus the elements of $C(R)$ are 'formal consequences'

$$
c=\prod_{i=1}^{n}\left(r_{i}^{\varepsilon_{i}}\right)^{u_{i}}
$$

where $n \geqslant 0, r_{i} \in R, \epsilon_{i}= \pm 1, u_{i} \in F(X), \delta_{2}\left(r^{\varepsilon}\right)^{u}=u^{-1}(\omega r)^{\varepsilon} u$, subject to the crossed module rule $a b=b a^{\delta_{2} b}, a, b \in C(R)$. For information on crossed modules, and particularly free crossed modules, see for example $[40,17,31,7]$.

Let $N=\operatorname{Ker} \varphi$. Then $\delta_{2}(C(R))=N$. Of course, it is the kernel $\pi(\mathcal{P})$ of $\delta_{2}$, the $G$-module of identities among relations, that we wish to calculate. For this we require algebraic analogues of methods of covering spaces, and so use the language of groupoids. Our convention is that the product of elements (arrows) $a: g \rightarrow g^{\prime}, a^{\prime}: g^{\prime} \rightarrow g^{\prime \prime}$ in a groupoid $\Gamma$ is written $a a^{\prime}: g \rightarrow g^{\prime \prime}$, and $\Gamma(g)$ denotes the object group of $\Gamma$ at $g$, that is the set of arrows $g \rightarrow g$ with the induced group structure.

2.3. $p_{0}: \widetilde{G} \rightarrow G$ is the universal covering groupoid of the group $G$. The objects of $\widetilde{G}$ are the elements of $G$, and an arrow of $\widetilde{G}$ is a pair $\left(g, g^{\prime}\right) \in G \times G$ with source $g=\delta^{0}\left(g, g^{\prime}\right)$ and target $g g^{\prime}=\delta^{1}\left(g, g^{\prime}\right)$. The projection morphism $p_{0}$ is given by $\left(g, g^{\prime}\right) \mapsto g^{\prime}$.

2.4. $\tilde{X}$ is the Cayley graph of the pair $(G, X)$. Its objects are the elements of $G$ and its arrows are pairs $(g, x) \in G \times X$ with source $g=\delta^{0}(g, x)$ and target $g(\varphi x)=\delta^{1}(g, x)$, also written $\beta(g, x)$.

2.5. $F(\tilde{X})$ is the free groupoid on $\tilde{X}$. Its objects are the elements of $G$ and its arrows are pairs $(g, u) \in G \times F(X)$ with source $g$ and target $g(\varphi u)$. We also write $\beta(g, u)=g(\varphi u)$. The multiplication is given by $(g, u)(g(\varphi u), v)=(g, u v)$. The morphism $\tilde{\varphi}$ is given by $(g, u) \mapsto(g, \varphi u)$. The morphism $p_{1}$ is given by $(g, u) \mapsto u$. It maps the object group $F(\widetilde{X})(1)$ isomorphically to $N$.

As we shall see in Section $7, \widetilde{G} \rightarrow G$ is the covering morphism corresponding to the trivial subgroup of $G$, and $F(\tilde{X}) \rightarrow F(X)$ is the covering morphism corresponding to the subgroup $N$ of $F(X)$. 
2.6. $\widetilde{R}=G \times R$ and $\widetilde{\delta}_{2}: C(\widetilde{R}) \rightarrow F(\widetilde{X})$ is the free crossed $F(\widetilde{X})$-module on $\widetilde{\omega}: \widetilde{R} \rightarrow$ $F(\widetilde{X}), \quad(g, r) \mapsto(g, \omega(r))$. Then $C(\widetilde{R})$ is the disjoint union of groups $C(\widetilde{R})(g), g \in G$, all mapped by $p_{2}$ isomorphically to $C(R)$. Elements of $C(\widetilde{R})(g)$ are pairs $(g, c) \in\{g\} \times$ $C(R)$, with multiplication $(g, c)\left(g, c^{\prime}\right)=\left(g, c c^{\prime}\right)$. The (partial) action of $F(\widetilde{X})$ is given by $(g, c)^{(g, u)}=\left(g(\varphi u), c^{u}\right)$. The boundary $\widetilde{\delta}_{2}$ is given by $(g, c) \mapsto\left(g, \delta_{2} c\right)$. The morphism $p_{2}: C(\widetilde{R}) \rightarrow C(R)$ is given by $(g, c) \mapsto c$.

If $(g, c) \in C(\widetilde{R})(g)$ we write $\beta(g, c)=g$; we call $\beta$ the base point map. The elements of $C(\widetilde{R})(g)$ are also all 'formal consequences'

$$
(g, c)=\prod_{i=1}^{n}\left(\left(g_{i}, r_{i}\right)^{\varepsilon_{i}}\right)^{\left(g_{i}, u_{i}\right)}=\prod_{i=1}^{n}\left(g,\left(r_{i}^{\varepsilon_{i}}\right)^{u_{i}}\right)=\left(g, \prod_{i=1}^{n}\left(r_{i}^{\varepsilon_{i}}\right)^{u_{i}}\right)
$$

where $n \geqslant 0, r_{i} \in R, \varepsilon_{i}= \pm 1, u_{i} \in F(X), g_{i} \in G, g_{i}\left(\varphi u_{i}\right)=g$, subject to the crossed module rule $a b=b a^{\widetilde{\delta}_{2} b}, a, b \in C(\widetilde{R})$. Here the first form of the product is useful geometrically, and the last is useful computationally.

In effect, we are giving first a presentation $\langle\widetilde{X} \mid \widetilde{\omega}: \widetilde{R} \rightarrow F(\widetilde{X})\rangle$ of the groupoid $\widetilde{G}$ [29], and second the free crossed module corresponding to this presentation.

The proof that the construction given in Paragraph 2.6 does give the free crossed module as claimed is given in Theorem 9.2.

We now construct $C_{3}(I)$ and its cover $C_{3}(\widetilde{I})$.

2.7. Let $I$ be a set in one-to-one correspondence with $G \times R$ with elements written [ $g, r], g \in G, r \in R$. Let $C_{3}(I)$ be the free $G$-module on $I$.

2.8. Let $C_{3}(\widetilde{I})$ be the free $\widetilde{G}$-module on $\widetilde{I}=G \times I$. Then $C_{3}(\widetilde{I})$ is the disjoint union of abelian groups $C(\widetilde{I})(g), g \in G$, all mapped by $p_{3}$ isomorphically to $C_{3}(I)$. Elements of $C_{3}(\widetilde{I})(g)$ are pairs $(g, i) \in\{g\} \times C_{3}(I)$ with addition $(g, i)+\left(g, i^{\prime}\right)=\left(g, i+i^{\prime}\right)$. The (partial) action of $\widetilde{G}$ on $C_{3}(\widetilde{I})$ is given by $(g, i) .\left(g, g^{\prime}\right)=\left(g g^{\prime}, i . g^{\prime}\right)$.

The construction of $\delta_{3}$ (and hence of $\widetilde{\delta}_{3}$ ) requires some choices.

2.9. Choose a section $\sigma: G \rightarrow F(X)$ of $\varphi$ such that $\sigma(1)=1$, and write $\bar{\sigma}(g)=\sigma(g)^{-1}$. Then $\sigma$ determines a function $h_{0}: G \rightarrow F(\widetilde{X})$ by $g \mapsto(g, \bar{\sigma} g)$. Thus $h_{0}(g)$ is a path $g \rightarrow 1$ in the Cayley graph $\widetilde{X}$.

Remark 2.1. The choice of $h_{0}$ is often, but not always, made by choosing a maximal tree in the graph $\tilde{X}$ - such a choice is equivalent to a choice of Schreier transversal for the subgroup $N=\operatorname{Ker} \varphi$ of $F(X)$.

For each arrow $(g, x)$ of $\tilde{X}$, the element $\rho(g, x)=\left(h_{0} g\right)^{-1}(g, x) h_{0}(g(\varphi x))$ is a loop at 1 in $F(\tilde{X})$, and so is in the image of $\tilde{\delta}_{2}$.

2.10. For each arrow $(g, x)$ of $\widetilde{X}$ choose an element $h_{1}(g, x) \in C(\widetilde{R})(1)$ such that

$$
\widetilde{\delta}_{2}\left(h_{1}(g, x)\right)=\rho(g, x) .
$$

Then $h_{1}$ extends uniquely to a morphism $h_{1}: F(\widetilde{X}) \rightarrow C(\widetilde{R})(1)$ such that for all arrows $(g, u)$ of $F(\tilde{X})$

$$
\widetilde{\delta}_{2}\left(h_{1}(g, u)\right)=\left(h_{0} g\right)^{-1}(g, u) h_{0}(g(\varphi u)) \text {. }
$$


It follows that $\tilde{\delta}_{2} h_{1}\left(h_{0}(g)\right)=(1,1)$ for all $g \in G$. Further, if $h_{0}$ is determined by a choice of maximal tree $T$ in the Cayley graph, then for each $(g, x)$ in $T$ we may choose $h_{1}(g, x)=(1,1)$.

Remark 2.2. The choice of $h_{1}$ is equivalent to choosing a representation as a consequence of the relators $R$ for each element of $N$, given as a word in the elements of $X$. There is no algorithm for such a choice. It will be shown in [28] how a 'logged Knuth-Bendix procedure' will give such a choice when the monoid rewrite system determined by $R$ may be completed, and that this allows for an implementation of the determination of $h_{1}$.

The morphism $k_{1}$ of Theorem 1.1 of the Introduction is simply the composition $p_{2} h_{1}$.

2.11. For $[g, r] \in I$ define

$$
\delta_{3}[g, r]=p_{2}\left(\left(h_{1}(g, \omega(r))\right)^{-1}\right) r^{\bar{\sigma} g} .
$$

It follows from equation (2) that $\delta_{2} \delta_{3}[g, r]=1$, and so the given values $\delta_{3}[g, r]$ lie in the $G$-module $\pi(\mathcal{P})$. This implies that $\delta_{3}: C_{3}(I) \rightarrow C(R)$ is well defined on $C_{3}(I)$ by its values on the set $I$ of free module generators.

2.12. Let $\widetilde{\delta}_{3}: C_{3}(\widetilde{I}) \rightarrow C(\widetilde{R})$ be the $\widetilde{G}$-morphism given by $\widetilde{\delta}_{3}(g, d)=\left(g, \delta_{3} d\right), d \in$ $C_{3}(I)$. Let $h_{2}: C(\widetilde{R}) \rightarrow C_{3}(\widetilde{I})(1)$ be the groupoid morphism killing the operation of $F(\tilde{X})$ (that is, $h_{2}\left((g, c)^{(g, u)}\right)=h_{2}(g, c)$ for all $\left.(g, c) \in C(\widetilde{R}), u \in F(X)\right)$ and satisfying $(g, r) \mapsto(1,[g, r]),(g, r) \in G \times R$. Then from equation (3) we deduce that for all $g \in G, c \in C(R)$

$$
\widetilde{\delta}_{3} h_{2}(g, c)=\left(h_{1} \widetilde{\delta}_{2}(g, c)\right)^{-1}\left(1, c^{\bar{\sigma} g}\right)
$$

2.13. Proof of Theorem 1.1. Equations (2) and (4) show that $\widetilde{\delta}_{2} \widetilde{\delta}_{3}=0$, and so the elements $p_{2}\left(\widetilde{\delta}_{3} h_{2}(g, c)\right)$ do give identities. On the other hand, if $c \in C(R)$ and $\delta_{2} c=1$, then by equation (4), $(1, c)=\widetilde{\delta}_{3} h_{2}(1, c)$, and so $c=\delta_{3}(d)$ for some $d$. Theorem 1.1 of the Introduction is an immediate consequence, with $k_{1}=p_{2} h_{1}$.

However some of the elements of $\delta_{3}(I)$ may be trivial, and others may depend $\mathbb{Z} G$ linearly on a smaller subset. That is, there may be a proper subset $J$ of $I$ such that $\delta_{3}(J)$ also generates the module $\pi(\mathcal{P})$. Then for each element $i \in I \backslash J$ there is a formula expressing $\delta_{3} i$ as a $\mathbb{Z} G$-linear combination of the elements of $\delta_{3}(J)$. These formulae determine a $\mathbb{Z} G$ retraction $\mathrm{r}: C_{3}(I) \rightarrow C_{3}(J)$ such that for all $d \in C_{3}(I), \delta_{3}(\mathrm{r} d)=\delta_{3}(d)$. So we replace $I$ in the above diagram by $J$, replacing the boundaries by their restrictions. Further, and this is the crucial step, we replace $h_{2}$ by $h_{2}^{\prime}=r^{\prime} h_{2}$ where $r^{\prime}: C_{3}(\widetilde{I})(1) \rightarrow C_{3}(\widetilde{J})(1)$ is mapped by $p_{3}$ to $r$.

This $h_{2}^{\prime}: C(\widetilde{R}) \rightarrow C_{3}(\widetilde{J})(1)$ is now used to continue the above construction, by defining $C_{4}(\bar{J})$ to be the free $G$-module on elements written $[g, d] \in \bar{J}=G \times J$, with

$$
\delta_{4}[g, d]=-p_{3}\left(h_{2}^{\prime}\left(g, \delta_{3} d\right)\right)+d \cdot g^{-1} .
$$

These boundary elements give generators for the relations among the generators $\delta_{3}(J)$ of $\pi(\mathcal{P})$.

2.14. Proof of Theorem 1.2. This is a similar argument to the proof of Theorem 1.1, using equation (5), and setting $k_{2}=p_{3} h_{2}^{\prime}$. 
Remark 2.3. In the discussion above we have defined morphisms and homotopies by their values on certain generators, and so it is important for this that the structures be free. For example, $h_{2}^{\prime}$ is defined by its values on the elements $(g, r) \in G \times R$. So, noting that $h_{2}$ kills the operation of $F(\tilde{X})$, we calculate for example $h_{2}^{\prime}\left(g, r^{u} s^{v}\right)=h_{2}^{\prime}\left(g(\varphi u)^{-1}, r\right)+$ $h_{2}^{\prime}\left(g(\varphi v)^{-1}, s\right)$. In this way the formulae reflect the choices made at different parts of the Cayley graph in order to obtain a contraction.

The freeness of $F(\tilde{X})$ was proved by Higgins in [29]. Our proof for $C(\widetilde{R})$ uses a result of Howie, as we shall see later.

Remark 2.4. The determination of minimal subsets $J$ of $I$ such that $\delta_{3} J$ also generates $\pi(\mathcal{P})$ is again not straightforward. Some dependencies are easy to find, and others are not. A basic result due to Whitehead [40] is that the abelianisation map $C(R) \rightarrow(\mathbb{Z} G)^{R}$ maps $\pi(\mathcal{P})$ isomorphically to the kernel of the Whitehead-Fox derivative $(\partial r / \partial x):(\mathbb{Z} G)^{R} \rightarrow$ $(\mathbb{Z} G)^{X}$. Hence we can test for dependency among identities by passing to the free $\mathbb{Z} G$ module $(\mathbb{Z} G)^{R}$, and we use this in the next section. For bigger examples, this testing can be a formidable task by hand. An implementation of Gröbner basis procedures for finding minimal subsets which still generate is described in [27].

We finally note in this section that in many cases the elements given by Theorem 1.1 form an additive generating set. This was suggested by the referee.

Theorem 2.5. Suppose that the function $h_{0}$ is determined by a choice of tree in the Cayley graph. Then the elements $\delta_{3}[g, r],(g, r) \in G \times R$ of Theorem 1.1 form an additive set of generators of $\pi(\mathcal{P})$.

Proof. We recall a commutative diagram due to Whitehead [40, Lemma 8], which is explored in the situation of general crossed complexes of groupoids in [15]:

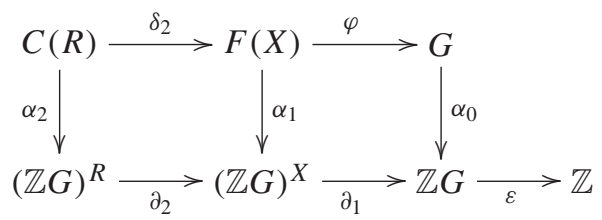

Here $\alpha_{2}$ is effectively abelianisation; $\alpha_{1}$ is the universal $\varphi$-derivation [22], and is defined on the free basis $X$ by $x \mapsto x$; $\alpha_{0}$ is the derivation $g \mapsto g-1$. Whitehead proves that these conditions imply that $\partial_{2}$ is well-defined and is a $G$-morphism. Then the derivation property implies that $\partial_{2}$ is the differential $(\partial r / \partial x)$ of the Fox free differential calculus. (We call the differential the 'Whitehead-Fox derivative' because Whitehead's paper was published four years before the more widely referenced paper of Fox [23].) It follows from the fundamental exact sequence of [22] (see also [15]) that the image of $\partial_{2}$ is $N^{\mathrm{ab}}$, the abelianisation of the kernel $N$ of $\varphi$.

Let $T$ be the chosen tree in $\tilde{X}$, and let $D$ be the set of edges of $\tilde{X}$ not in $T$. The tree determines a retraction $\rho: F(\widetilde{X}) \rightarrow F(\widetilde{X})(1)$, and by standard Schreier theory the set $p_{1} \rho(D)$ freely generates $N$. Define a morphism $s: N \rightarrow C(R)$ by $s\left(p_{1} \rho(\theta)\right)=k_{1}(\theta), \theta \in$ $D$. By the conditions on $k_{1}$, we have $\delta_{2} s=1_{N}$. This section $s$ of the restriction of $\delta_{2}$ yields an additive section $s^{\prime}: N^{\mathrm{ab}} \rightarrow(\mathbb{Z} G)^{R}$ of the restriction of $\partial_{2}$.

Let $(g, r) \in \widetilde{R}$. Then $k_{1}(g, \omega r)=k_{1} \rho(g, \omega r)$. If $\rho(g, \omega r)=\left(\rho \theta_{1}\right) \ldots \rho\left(\theta_{t}\right), \theta_{i} \in D$, 
then

$$
\begin{aligned}
k_{1}(g, \omega r) & =k_{1} \rho(g, \omega r) \\
& =\left(k_{1} \rho \theta_{1}\right) \ldots\left(k_{1} \rho \theta_{t}\right) \\
& =\left(s p_{1} \rho \theta_{1}\right) \ldots\left(s p_{1} \rho \theta_{t}\right) \\
& =s p_{1}\left(\left(\rho \theta_{1}\right) \ldots\left(\rho \theta_{t}\right)\right) \\
& =s p_{1} \rho(g, \omega r) .
\end{aligned}
$$

It follows that

$$
\begin{aligned}
\alpha_{2} k_{1}(g, \omega r) & =\alpha_{2} s p_{1} \rho(g \omega r) \\
& =s^{\prime} \alpha_{1} p_{1} \rho(g, \omega r) \\
& =s^{\prime} \alpha_{1}((\sigma g)(\omega r)(\bar{\sigma} g)) \\
& =s^{\prime} \alpha_{1}\left(\delta_{2}\left(r^{\bar{\sigma} g}\right)\right) \\
& =s^{\prime} \partial_{2} \alpha_{2}\left(r^{\bar{\sigma} g}\right) \\
& =s^{\prime} \partial_{2}\left(r . g^{-1}\right) .
\end{aligned}
$$

The elements $r \cdot g^{-1}$ generate $(\mathbb{Z} G)^{R}$ additively and so the elements $r \cdot g^{-1}-s^{\prime} \partial_{2}\left(r \cdot g^{-1}\right)$ generate Ker $\partial_{2}$ additively. Hence the elements $\alpha_{2} \delta_{3}[g, r]$ generate Ker $\partial_{2}$ additively.

Remark 2.6. The construction of a section such as $s^{\prime}$ is a common technique. One of the points of our methods is that a computational description of $s^{\prime}$ is obtained from geometric information on the Cayley graph.

\section{Syzygies of levels 2 and 3 for the standard presentation of $S_{3}$}

We illustrate the above method in this section with the standard presentation of the sixelement group $S_{3}$. This is chosen as perhaps the first interesting example which can still be done by hand, and because it does illustrate all the above points. While our set of generators of the module of identities for this presentation is known, we are not aware of previous calculations of the relations between these generators.

The group presentation $\left\langle x, y \mid x^{3}, y^{2}, x y x y\right\rangle$ determines the symmetric group $S_{3}$ on three symbols. Let $X=\{x, y\}$ and let $F=F(X)$ be the free group on $X$. Let $R=\{r, s, t\}$ and let $\omega: R \rightarrow F$ be given by

$$
\omega r=x^{3}, \omega s=y^{2}, \omega t=x y x y .
$$

Let $\varphi: F \rightarrow S_{3}$ be the epimorphism determined by $x, y$, and let $N=\operatorname{Ker} \varphi$. So we have the free crossed module $\delta_{2}: C(R) \rightarrow F$.

Now we set up the corresponding diagram (1) of the previous section. We think of each element $(g, r) \in \widetilde{R}$ as filling a 2-cell in the Cayley graph $\widetilde{X}$. Thus, in this example, each relator, that is each element of $R$, is covered six times in the universal cover. We also see in this situation the rôle of relations which are proper powers. The covers of the element $r$ of $R$ separate into two classes, namely

$$
\left\{(1, r),(\varphi x, r),\left(\varphi x^{2}, r\right)\right\}, \quad\left\{(\varphi y, r),(\varphi y x, r),\left(\varphi y x^{2}, r\right)\right\} .
$$

An element of one of these classes has as its boundary the same 'triangle' in $F(\tilde{X})$ as the other elements, but with a different starting point. Similarly, the relation $\omega t$ is of order 2 


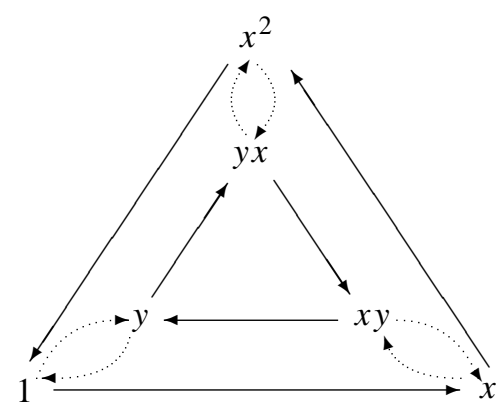

(a) The Cayley graph of $S_{3}$

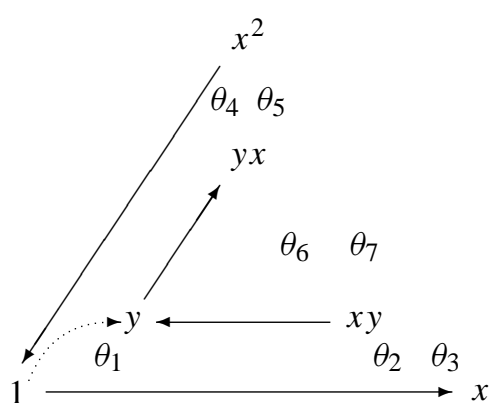

(b) A tree in it

Figure 1: The Cayley graph of $S_{3}$, and a tree in it

and so the covers of $t$ divide into three classes, each with 2 elements. A similar statement holds for $s$.

We now have to choose $\sigma: S_{3} \rightarrow F(X)$. For this, choose a maximal tree $T$ in the directed graph $\widetilde{X}$. The choice of $T$ is well known to be equivalent to the choice of a Schreier transversal for $N$ in $F$. For this example, we choose the tree $T$ to be given by the elements

$$
(1, y),(1, x),\left(\varphi x^{2}, x\right),(\varphi y, x),(\varphi(x y), x) .
$$

The remaining elements of $\widetilde{X}$ we label as

$$
\begin{gathered}
\theta_{1}=(\varphi y, y), \theta_{2}=(\varphi x, y), \theta_{3}=(\varphi x y, y), \theta_{4}=(\varphi y x, y), \\
\theta_{5}=\left(\varphi x^{2}, y\right), \theta_{6}=(\varphi y x, x), \theta_{7}=(\varphi x, x)
\end{gathered}
$$

The object groups of the free groupoid $F(T)$ on the graph $T$ are all trivial. For each $g \in S_{3}$ let $h_{0} g$ denote the unique element of $F(T)(g, 1)$, so that the section $\sigma: S_{3} \rightarrow F$ of $\varphi$ is given by $h_{0} g=\left(g,(\sigma g)^{-1}\right), g \in G$. Then for each $(g, u): g \rightarrow g^{\prime}$ in $F(\widetilde{X})$, we set $\rho(g, u)=\left(h_{0}(g)\right)^{-1}(g, u) h_{0}\left(g^{\prime}\right)$. Let $\widetilde{N}=F(\tilde{X})(1)$, which is mapped isomorphically by $p_{2}$ to $N=\delta_{2}(C(R))=\operatorname{Ker} \varphi$. Thus the tree $T$ determines a retraction $\rho: F(\widetilde{X}) \rightarrow \widetilde{N}$.

Let $D$ be the set of edges of $\widetilde{X}$ which do not lie in $T$. Then the set $\rho(D)$ is a set of free generators of the group $\widetilde{N}$, and $p_{1} \rho(D)$ is a set of free generators of the group $N$. Let $\eta_{i}=\rho \theta_{i}, i=1, \ldots, 7$.

In order to define $h_{1}: F(\widetilde{X}) \rightarrow C(\widetilde{R})(1)$ we need only to give its values on the generators (see Section 10.1). We give these by $h_{1}(\tau)=1$ if $\tau \in T$ and for $\theta \in D$, we let $h_{1}(\theta)$ be an element of $C(\widetilde{R})(1)$ which is mapped by $\widetilde{\delta}_{2}$ to $\rho(\theta)$. Then $h_{1}$ satisfies (2), and also $h_{1}(\theta)=h_{1}(\rho(\theta))$. 
Table 1: Eighteen generators for identities

\begin{tabular}{lllll}
\hline \multicolumn{2}{c}{ generator } & $\rho \tilde{\delta}_{2} \alpha_{i}$ & \multicolumn{1}{c}{$\gamma_{i}=p_{2}\left(h_{1}\left(\tilde{\delta}_{2} \alpha_{i}\right)^{-1} \alpha_{i}^{h_{0} \beta \alpha_{i}}\right)$} \\
\hline$\alpha_{1}$ & $\left(\varphi x^{2}, r\right)$ & $\eta_{7}$ & $\gamma_{1}$ & $r^{-1} r^{x}$ \\
$\alpha_{2}$ & $(\varphi y, s)$ & $\eta_{1}$ & $\gamma_{2}$ & $s^{-1} s^{y^{-1}}$ \\
$\alpha_{3}$ & $\left(\varphi x^{2}, s\right)$ & $\eta_{5} \eta_{4}$ & $\gamma_{3}$ & $\left(t^{-1}\right)^{y^{-1}} t^{x}$ \\
$\alpha_{4}$ & $(\varphi x, t)$ & $\eta_{7} \eta_{5} \eta_{6} \eta_{3}$ & $\gamma_{4}$ & $\left(s^{-1}\right)^{x^{-1}} t s^{-1}\left(r^{-1}\right)^{y^{-1}} t^{x}\left(s^{-1}\right)^{x} r^{-1} t^{x^{-1}}$ \\
$\alpha_{5}$ & $(1, r)$ & $\eta_{7}$ & $\gamma_{5}$ & 1 \\
$\alpha_{6}$ & $(1, s)$ & $\eta_{1}$ & $\gamma_{6}$ & 1 \\
$\alpha_{7}$ & $(1, t)$ & $\eta_{2} \eta_{1}$ & $\gamma_{7}$ & 1 \\
$\alpha_{8}$ & $(\varphi x, s)$ & $\eta_{2} \eta_{3}$ & $\gamma_{8}$ & 1 \\
$\alpha_{9}$ & $(\varphi y, t)$ & $\eta_{5}$ & $\gamma_{9}$ & 1 \\
$\alpha_{10}$ & $(\varphi y, r)$ & $\eta_{6}$ & $\gamma_{10}$ & 1 \\
$\alpha_{11}$ & $(\varphi x, r)$ & $\eta_{7}$ & $\gamma_{11}$ & $r^{-1} r^{x^{-1}}$ \\
$\alpha_{12}$ & $(\varphi x y, r)$ & $\eta_{6}$ & $\gamma_{12}$ & $\left(r^{-1}\right)^{y^{-1}} r^{x y^{-1}}$ \\
$\alpha_{13}$ & $(\varphi y x, r)$ & $\eta_{6}$ & $\gamma_{13}$ & $\left(r^{-1}\right)^{y^{-1}} r^{x^{-1} y^{-1}}$ \\
$\alpha_{14}$ & $(\varphi x y, s)$ & $\eta_{3} \eta_{2}$ & $\gamma_{14}$ & $\left(s^{-1}\right)^{y x y^{-1}} s^{x y^{-1}}$ \\
$\alpha_{15}$ & $(\varphi x y, t)$ & $\eta_{1} \eta_{2}$ & $\gamma_{15}$ & $\left(t^{-1}\right)^{y^{-2}} t^{x y^{-1}}$ \\
$\alpha_{16}$ & $\left(\varphi x x^{2}, t\right)$ & $\eta_{5}$ & $\gamma_{16}$ & $\left(t^{-1}\right)^{y^{-1}} t^{x}$ \\
$\alpha_{17}$ & $(\varphi y x, s)$ & $\eta_{4} \eta_{5}$ & $\gamma_{17}$ & $t^{x}\left(s^{-1}\right)^{x}\left(t^{-1}\right)^{y^{-1}} s^{x^{-1} y^{-1}}$ \\
$\alpha_{18}$ & $(\varphi y x, t)$ & $\eta_{6} \eta_{3} \eta_{7} \eta_{5}$ & $\gamma_{18}$ & $t^{x}\left(s^{-1}\right)^{x} r^{-1}\left(s^{-1}\right)^{x^{-1}} t s^{-1}\left(r^{-1}\right)^{y^{-1}} t^{x^{-1} y^{-1}}$ \\
\hline
\end{tabular}

In our example of $S_{3}$, we define $h_{1}$ on $\rho(D)$, and so on $F(\tilde{X})$, as follows:

$$
\begin{aligned}
& \eta_{1}=\rho(\varphi y, y), \quad h_{1} \eta_{1}=(1, s) \quad=(1, s) \\
& \eta_{2}=\rho(\varphi x, y), \quad h_{1} \eta_{2}=(1, t)(1, s)^{-1} \quad=\left(1, t s^{-1}\right) \\
& \eta_{3}=\rho(\varphi x y, y), \quad h_{1} \eta_{3}=(1, s)(1, t)^{-1}(\varphi x, s)^{(1, x)^{-1}} \quad=\left(1, s t^{-1} s^{x^{-1}}\right) \\
& \eta_{4}=\rho(\varphi y x, y), \quad h_{1} \eta_{4}=(\varphi y, t)^{(1, y)^{-1}}=\left(1, t^{y^{-1}}\right) \\
& \eta_{5}=\rho\left(\varphi x^{2}, y\right), \quad h_{1} \eta_{5}=\left(\varphi x^{2}, s\right)^{\left(\varphi x^{2}, x\right)}\left(\left(\varphi x^{2}, t\right)^{-1}\right)^{\left(\varphi x^{2}, x\right)}=\left(1, s^{x}\left(t^{-1}\right)^{x}\right) \\
& \eta_{6}=\rho(\varphi y x, x), \quad h_{1} \eta_{6}=(\varphi y, r)^{(1, y)^{-1}}=\left(1, r^{y^{-1}}\right) \\
& \eta_{7}=\rho(\varphi x, x), \quad h_{1} \eta_{7}=(1, r) \quad=(1, r)
\end{aligned}
$$

This ensures that $\widetilde{\delta}_{2} h_{1}\left(\eta_{i}\right)=\eta_{i}, i=1,2, \ldots, 7$.

In order to calculate the identities among relations, we now need to express $\rho \widetilde{\delta}_{2} \alpha$ in terms of the $\eta_{i}$ for all $\alpha \in \widetilde{R}$. Then according to the previous section we can obtain an identity among relations for each $\alpha \in \widetilde{R}$, namely

$$
p_{2}\left(\left(h_{1} \widetilde{\delta}_{2} \alpha\right)^{-1} \alpha^{h_{0} \beta \alpha}\right) .
$$

The results of these calculations are given in Table 1. The order of writing the identities is chosen so that the first four give our eventual minimal set of generators, the next six give trivial identities, and the last has the most difficult verification of its dependence on the first four. 
Table 2: Reduction to four identities

\begin{tabular}{|c|c|c|}
\hline$\gamma_{i}$ & identity & $r \bar{\alpha}_{i}$ \\
\hline$\gamma_{11}$ & $=\left(\gamma_{1}^{-1}\right)^{x^{-1}}$ & $-\bar{\alpha}_{1} \cdot x^{2}$ \\
\hline$\gamma_{12}$ & $=\gamma_{1}^{y^{-1}}$ & $\bar{\alpha}_{1} \cdot y$ \\
\hline$\gamma_{13}$ & $=\left(\gamma_{1}^{-1}\right)^{x^{-1} y^{-1}}$ & $-\bar{\alpha}_{1} \cdot y x$ \\
\hline$\gamma_{14}$ & $=\gamma_{2}^{y x y^{-1}}$ & $\bar{\alpha}_{2} \cdot x^{2}$ \\
\hline$\gamma_{15}$ & $=\gamma_{3}^{y^{-1}}$ & $\bar{\alpha}_{3} \cdot y$ \\
\hline$\gamma_{16}$ & $=\gamma_{3}$ & $\bar{\alpha}_{3}$ \\
\hline$\gamma_{17}$ & $=\gamma_{3}\left(\gamma_{2}^{-1}\right)^{x^{-1} y^{-1}}$ & $\bar{\alpha}_{3}-\bar{\alpha}_{2} \cdot y x$ \\
\hline$\gamma_{18}$ & $=\gamma_{4} \gamma_{3}^{y x^{2}}$ & $\bar{\alpha}_{4}+\bar{\alpha}_{3} . x y$ \\
\hline
\end{tabular}

We now let $I$ consist of elements $\bar{\alpha}_{i}$ in one-to-one correspondence with the $\alpha_{i}$, and let $C_{3}(I)$ be the free $G$-module on $I$. Define $\delta_{3} \bar{\alpha}_{i}$ to be the value $\gamma_{i} \in C_{2}$ given in the fourth column of the table. Let $\widetilde{I}=G \times I$, and let $C_{3}(\widetilde{I})$ be the free $\widetilde{G}$-module on $\widetilde{I}$. We define

$$
h_{2}\left(\alpha_{i}\right)=\left(1, \bar{\alpha}_{i}\right), i=1, \ldots, 18 .
$$

Then we have

$$
h_{1}\left(\widetilde{\delta}_{2} \alpha_{i}\right)^{-1} \alpha_{i}^{h_{0} \beta\left(\alpha_{i}\right)}=\left(1, \gamma_{i}\right)=\widetilde{\delta}_{3} h_{2} \alpha_{i}, i=1, \ldots, 18
$$

So we have extended our covering complex and its contracting homotopy by one stage.

However, we can in fact omit all of the $\alpha_{i}$ except the first four, because of the trivial identities $\gamma_{5}, \ldots, \gamma_{10}$, and the further relations given in Table 2 . We give the verification for the last two further relations, the others being trivial or easy.

We note that

$$
\begin{aligned}
& \gamma_{17}=t^{x}\left(s^{-1}\right)^{x}\left(t^{-1}\right)^{y^{-1}} s^{x^{-1} y^{-1}} \\
& =t^{x}\left(t^{-1}\right)^{y^{-1}}\left(s^{-1}\right)^{x y y^{-1} x^{-1} y^{-1} x^{-1} y^{-1}} s^{x^{-1} y^{-1}} \\
& =\left(t^{-1}\right)^{y^{-1}} t^{y^{-1} x^{-1} y^{-1}}\left(\left(s^{-1}\right)^{y^{-1}} s\right)^{x^{-1} y^{-1}} \\
& =\left(t^{-1}\right)^{y^{-1}} t^{x}\left(\left(s^{-1}\right)^{y^{-1}} s\right)^{x^{-1} y^{-1}} \\
& =\gamma_{3}\left(\gamma_{2}^{-1}\right)^{x^{-1} y^{-1}} \text {. }
\end{aligned}
$$

by the crossed module rules since $t^{\left(\delta_{2} t^{-1}\right) x}=t^{x}$

In order to verify the further identity for $\gamma_{18}$, we consider the abelianisation $C(R)^{\mathrm{ab}}$, which is isomorphic to the free $S_{3}$-module on $R$. The difference $\gamma_{18}-\gamma_{4}$ in $C(R)^{\mathrm{ab}}$ is

$$
t \cdot\left(\varphi\left(x^{-1} y^{-1}\right)-\varphi\left(x^{-1}\right)\right)=t .(\varphi(x y)-1) \varphi\left(x^{2}\right) .
$$

Since the module of identities is mapped injectively into $C(R)^{\mathrm{ab}}$ [17], and in $C(R)^{\mathrm{ab}}$ we have $\gamma_{3}=t .\left(\varphi(x)-\varphi\left(y^{-1}\right)\right)$, the result follows. So we have a set of four generators for the module of identities for this presentation of $S_{3}$, of which the first three given belong to the root module (see [17] for an account of this).

Let $J$ consist of the elements $\bar{\alpha}_{i}, i=1, \ldots, 4$, and let $C_{3}(J)$ be the free $G$-module on $J$, with the restriction to it of the boundary $\delta_{3}$. Let $r: C_{3}(I) \rightarrow C_{3}(J)$ be be the $G$-module morphism defined by $\mathrm{r}\left(\bar{\alpha}_{i}\right)=\bar{\alpha}_{i}, i=1, \ldots, 4, \mathrm{r}\left(\bar{\alpha}_{i}\right)=0, i=5, \ldots, 10$ and otherwise as in Table 2, so that $\delta_{3} r=\delta_{3}$. Note that $C_{3}(I)$ is an $S_{3}$-module, and so we write it additively 
Table 3: Twenty-four generators for relations among identities

\begin{tabular}{|c|c|c|c|}
\hline \multicolumn{2}{|c|}{ generator } & \multicolumn{2}{|r|}{ identity $=p_{3}\left(-h_{2}^{\prime} \tilde{\delta}_{3} \theta_{i}+\theta_{i}^{h_{0} \beta \theta_{i}}\right)$} \\
\hline$\xi_{1}$ & $\left(1, \bar{\alpha}_{1}\right)$ & $\mu_{1}$ & 0 \\
\hline$\xi_{2}$ & $\left(1, \bar{\alpha}_{2}\right)$ & $\mu_{2}$ & 0 \\
\hline$\xi_{3}$ & $\left(1, \bar{\alpha}_{3}\right)$ & $\mu_{3}$ & 0 \\
\hline$\xi_{4}$ & $\left(1, \bar{\alpha}_{4}\right)$ & $\mu_{4}$ & 0 \\
\hline$\xi_{5}$ & $\left(\varphi x, \bar{\alpha}_{1}\right)$ & $\mu_{5}$ & 0 \\
\hline$\xi_{6}$ & $\left(\varphi x, \bar{\alpha}_{2}\right)$ & $\mu_{6}$ & 0 \\
\hline$\xi_{7}$ & $\left(\varphi x, \bar{\alpha}_{3}\right)$ & $\mu_{7}$ & $\bar{\alpha}_{3} \cdot\left(y+x^{2}\right)$ \\
\hline$\xi_{8}$ & $\left(\varphi x, \bar{\alpha}_{4}\right)$ & $\mu_{8}$ & $\bar{\alpha}_{1} \cdot\left(y-x^{2}\right)+\bar{\alpha}_{4} \cdot\left(x^{2}-1\right)$ \\
\hline$\xi_{9}$ & $\left(\varphi x^{2}, \bar{\alpha}_{1}\right)$ & $\mu_{9}$ & $\bar{\alpha}_{1} \cdot\left(1+x+x^{2}\right)$ \\
\hline$\xi_{10}$ & $\left(\varphi x^{2}, \bar{\alpha}_{2}\right)$ & $\mu_{10}$ & $\bar{\alpha}_{2} \cdot(1+y) x$ \\
\hline$\xi_{11}$ & $\left(\varphi x^{2}, \bar{\alpha}_{3}\right)$ & $\mu_{11}$ & $\bar{\alpha}_{3} \cdot(1+y x) x$ \\
\hline$\xi_{12}$ & $\left(\varphi x^{2}, \bar{\alpha}_{4}\right)$ & $\mu_{12}$ & $\bar{\alpha}_{1} \cdot(1-y x)+\bar{\alpha}_{4} \cdot(x-1)$ \\
\hline$\xi_{13}$ & $\left(\varphi y, \bar{\alpha}_{1}\right)$ & $\mu_{13}$ & 0 \\
\hline$\xi_{14}$ & $\left(\varphi y, \bar{\alpha}_{2}\right)$ & $\mu_{14}$ & $\bar{\alpha}_{2} \cdot(1+y)$ \\
\hline$\xi_{15}$ & $\left(\varphi y, \bar{\alpha}_{3}\right)$ & $\mu_{15}$ & 0 \\
\hline$\xi_{16}$ & $\left(\varphi y, \bar{\alpha}_{4}\right)$ & $\mu_{16}$ & $\bar{\alpha}_{2} \cdot\left(1+x^{2}-y x\right)+\bar{\alpha}_{3} \cdot(1-y-x y)-\bar{\alpha}_{4} \cdot(1-y)$ \\
\hline$\xi_{17}$ & $\left(\varphi y x, \bar{\alpha}_{1}\right)$ & $\mu_{17}$ & 0 \\
\hline$\xi_{18}$ & $\left(\varphi y x, \bar{\alpha}_{2}\right)$ & $\mu_{18}$ & 0 \\
\hline$\xi_{19}$ & $\left(\varphi y x, \bar{\alpha}_{3}\right)$ & $\mu_{19}$ & $\bar{\alpha}_{3} \cdot(1+y x)$ \\
\hline$\xi_{20}$ & $\left(\varphi y x, \bar{\alpha}_{4}\right)$ & $\mu_{20}$ & $\begin{array}{r}\bar{\alpha}_{1} \cdot(1-y x)+\bar{\alpha}_{2} \cdot\left(1+x^{2}-y x\right)+\bar{\alpha}_{3} \cdot(1-y-x y)+ \\
+\bar{\alpha}_{4} \cdot(-1+y x)\end{array}$ \\
\hline$\xi_{21}$ & $\left(\varphi y x^{2}, \bar{\alpha}_{1}\right)$ & $\mu_{21}$ & $\bar{\alpha}_{1} \cdot\left(1+x+x^{2}\right) y$ \\
\hline$\xi_{22}$ & $\left(\varphi y x^{2}, \bar{\alpha}_{2}\right)$ & $\mu_{22}$ & $\bar{\alpha}_{2} \cdot(1+y) x^{2}$ \\
\hline$\xi_{23}$ & $\left(\varphi y x^{2}, \bar{\alpha}_{3}\right)$ & $\mu_{23}$ & 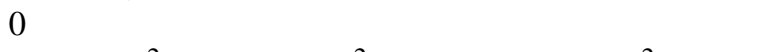 \\
\hline$\xi_{24}$ & $\left(\varphi y x^{2}, \bar{\alpha}_{4}\right)$ & $\mu_{24}$ & $\begin{array}{r}\bar{\alpha}_{1} \cdot\left(y-x^{2}\right)+\bar{\alpha}_{2} \cdot\left(1+x^{2}-y x\right)+\bar{\alpha}_{3} \cdot\left(1+x^{2}-x y\right)+ \\
+\bar{\alpha}_{4} \cdot(-1+x y)\end{array}$ \\
\hline
\end{tabular}

as a group, and use '? for the group action. To simplify the notation we write these acting elements as words in the generators $x, y$.

Define $\mathrm{r}^{\prime}: C_{3}(\widetilde{I}) \rightarrow C_{3}(\widetilde{J}),(g, d) \mapsto(g, \mathrm{r} d)$, and define $h_{2}^{\prime}=\mathrm{r}^{\prime} h_{2}: C(\widetilde{R}) \rightarrow C_{3}(\widetilde{J})$. Then we have for $i=1,2, \ldots, 18$

$$
\widetilde{\delta}_{3} h_{2}^{\prime} \alpha_{i}=h_{1}\left(\widetilde{\delta}_{2} \alpha_{i}\right)^{-1} \alpha_{i}^{h_{0} \beta \alpha_{i}}
$$

and so we have a contracting homotopy up to this level.

Note that we now have 24 generators $\tilde{d}=\left(g, \bar{\alpha}_{i}\right), g \in S_{3}, i=1, \ldots, 4$ of $C_{3}(\tilde{J})$ and we can proceed to the next stage, to obtain identities between identities corresponding to each of these generators, namely

$$
p_{3}\left(-h_{2}^{\prime} \tilde{\delta}_{3} \tilde{d}+\tilde{d}^{h_{0} \beta \tilde{d}}\right)
$$

for $\tilde{d}=\left(g, \bar{\alpha}_{i}\right), g \in S_{3}, i=1, \ldots, 4$. This requires another table (Table 3$)$. In order to show how the calculations go, we next carry out one intermediate calculation, and one full calculation. Further details of the calculations required for the table are omitted, but are available on request. 
Table 4: Reduction to five relations among identities

\begin{tabular}{ll}
\hline generator & definition / further identity \\
\hline$\mu_{9}$ & $\bar{\alpha}_{1} \cdot\left(1+x+x^{2}\right)$ \\
$\mu_{14}$ & $\bar{\alpha}_{2} \cdot(1+y)$ \\
$\mu_{19}$ & $\bar{\alpha}_{3} \cdot(1+y x)$ \\
$\mu_{12}$ & $\bar{\alpha}_{1} \cdot(1-y x)+\bar{\alpha}_{4} \cdot(x-1)$ \\
$\mu_{16}$ & $\bar{\alpha}_{2} \cdot\left(1+x^{2}-y x\right)+\bar{\alpha}_{3} \cdot(1-y-x y)-\bar{\alpha}_{4} \cdot(1-y)$ \\
$\mu_{21}$ & $=\mu_{9} \cdot y$ \\
$\mu_{10}$ & $=\mu_{14} \cdot x$ \\
$\mu_{22}$ & $=\mu_{14} \cdot x^{2}$ \\
$\mu_{11}$ & $=\mu_{19} \cdot x$ \\
$\mu_{7}$ & $=\mu_{19} \cdot x^{2}$ \\
$\mu_{8}$ & $=\mu_{9} \cdot(-1+y)+\mu_{12} \cdot(x+1)$ \\
$\mu_{20}$ & $=\mu_{9} \cdot(1-y)+\mu_{12} \cdot(x+1) y+\mu_{16}$ \\
$\mu_{24}$ & $=\mu_{12} \cdot y+\mu_{16}+\mu_{19} \cdot x^{2}$ \\
\hline
\end{tabular}

Recall that $h_{2}^{\prime}$ is a groupoid morphism, and kills the action of $F(\tilde{X})$. So, for example,

$$
\begin{aligned}
h_{2}^{\prime}\left(\varphi y, t^{x}\right) & =h_{2}^{\prime}\left(\left(\varphi y x^{-1}, t\right)^{(\varphi x y, x)}\right) \\
& =h_{2}^{\prime}(\varphi x y, t) \\
& =h_{2}^{\prime}\left(\alpha_{15}\right) \\
& =\left(1, \bar{\alpha}_{3}^{y^{-1}}\right) \\
& =\left(1, \bar{\alpha}_{3}^{\varphi y^{-1}}\right) \\
& =\left(1, \bar{\alpha}_{3}^{y}\right) .
\end{aligned}
$$

So we have

$$
\begin{aligned}
& -h_{2}^{\prime} \tilde{\delta}_{3}\left(\varphi y x, \bar{\alpha}_{4}\right)+\left(\varphi y x, \bar{\alpha}_{4}\right)^{\left(y x, x^{-1} y^{-1}\right)} \\
& =-h_{2}^{\prime}\left(\varphi y x,\left(s^{-1}\right)^{x^{-1}} t s^{-1}\left(r^{-1}\right)^{y^{-1}} t^{x}\left(s^{-1}\right)^{x} r^{-1} t^{x^{-1}}\right)+\left(1, \bar{\alpha}_{4}^{x^{-1} y^{-1}}\right) \\
& =-\left(1,-\bar{\alpha}_{2} \cdot x^{2}+\bar{\alpha}_{4}+\bar{\alpha}_{3} \cdot x y-\bar{\alpha}_{3}+\bar{\alpha}_{2} \cdot y x-\bar{\alpha}_{1}-\bar{\alpha}_{2}+\bar{\alpha}_{1} \cdot y x+\bar{\alpha}_{3} \cdot y-\bar{\alpha}_{4} \cdot y x\right) \\
& =\left(1, \bar{\alpha}_{1} \cdot(1-y x)+\bar{\alpha}_{2} \cdot\left(1+x^{2}-y x\right)+\bar{\alpha}_{3} \cdot(1-y-x y)+\bar{\alpha}_{4} \cdot(-1+y x)\right) .
\end{aligned}
$$

Some of the identities in Table 3 might seem as surprising to others as they were to the authors. There is a process for checking that these are identities among identities as follows.

We are required to check that $\delta_{3}$ of some combination $u$ of the $\bar{\alpha}_{i}$ is zero. Certainly, each $\delta_{3} \bar{\alpha}_{i}$ is an identity among relations, and hence so is the corresponding linear combination $u$. Therefore $u$ is 0 if and only if it maps to 0 in the abelianised group $C(R)^{\mathrm{ab}}$, which is freely generated as a $\mathbb{Z} S_{3}$ module by the elements $r, s, t$. Thus we determine the coefficients of these elements for the image of $u$ in $C(R)^{\mathrm{ab}}$, and it is straightforward to check that these are zero. This is analogous to a previous calculation.

We next reduce this to a smaller set of identities among identities, as in Table 4.

Let $K$ be the set with elements $\bar{\mu}_{9}, \bar{\mu}_{14}, \bar{\mu}_{19}, \bar{\mu}_{12}, \bar{\mu}_{16}$, let $C_{4}(K)$ be the free $G$-module on $K$, and let $\delta_{4}: C_{4}(K) \rightarrow C_{3}(J)$ be given by the first five lines of the second column of Table 4. Then the sequence $C_{4}(K) \rightarrow C_{3}(J) \rightarrow C(R)$ is exact and we have extended 
our crossed resolution by one further step. Hence we have a presentation of the $G$-module $\pi(\mathcal{P})$.

Such a crossed resolution has been extended, again by hand, by two further steps, but with different choices, in Example 5.5.1 of [26]. As stated in the Introduction, the numbers of generators in the next dimensions are found to be 6 and then 7 .

As explained in the Introduction, this example is chosen as one which illustrates the method, which has non-trivial calculations but also is perhaps the largest example of this type which one would care to do by hand. The major problems are: (i) the calculation of $h_{1}$ (that is, representing a set of group generators of $N=\operatorname{Ker} \varphi$ as consequences of the relators), and more seriously, (ii) finding minimal generating subsets of sets of generators of submodules of free $\mathbb{Z} G$-modules, as well as (iii) finding the relations giving all the generators in terms of the smaller set. The first problem is dealt with in [28], and the last two are dealt with in [27].

\section{Presentations of groupoids}

The category of groupoids will be written Gpd. Our convention for groupoids is that the composite of arrows $a: x \rightarrow y, b: y \rightarrow z$ is written $a b: x \rightarrow z$.

The theory of groupoids may be thought of as an algebraic analogue of the theory of groups, but based on directed graphs rather than on sets. For some discussion of the philosophy of this, see [5].

\subsection{Free groupoids}

The term graph will always mean what is usually called a directed graph. A graph $X$ consists of two sets $\operatorname{Arr}(X), \mathrm{Ob}(X)$, of arrows and objects respectively of $X$, and two functions $s, t: \operatorname{Arr}(X) \rightarrow \mathrm{Ob}(X)$, called the source and target maps. A morphism $f$ : $X \rightarrow Y$ of graphs consists of two functions $\operatorname{Arr}(X) \rightarrow \operatorname{Arr}(Y), \mathrm{Ob}(X) \rightarrow \mathrm{Ob}(Y)$, which commute with the source and target maps. This defines the category Graph.

A basic construction in any algebraic theory is that of free objects. For groups, the free group functor $F:$ Set $\rightarrow$ Group is left adjoint to the forgetful functor Group $\rightarrow$ Set. In the case of groupoids, we may define the free groupoid functor to be the left adjoint $F:$ Graph $\rightarrow$ Gpd to the forgetful functor $U:$ Gpd $\rightarrow$ Graph giving the underlying graph $U G$ of a groupoid $G$, namely forgetting the composition, the identity function $\operatorname{Ob}(G) \rightarrow G$, and the inverse map $G \rightarrow G$. So if $X$ is a graph, then the free groupoid $F(X)$ on $X$ consists of a graph morphism $i: X \rightarrow U F(X)$ which is universal for morphisms from $X$ to the underlying graph of a groupoid.

The set of objects of $F(X)$ may be identified with $\mathrm{Ob}(X)$. There are several ways of explicitly constructing the set of arrows of $F(X)$. The usual way is as equivalence classes of composable words

$$
w=\left(x_{1}, \varepsilon_{1}\right) \ldots\left(x_{n}, \varepsilon_{n}\right), n \geqslant 0, x_{i} \in \operatorname{Arr}(X), \varepsilon= \pm
$$

together with empty words ()$_{a}, a \in \mathrm{Ob}(X)$, where the word $w$ is composable means that $t\left(x_{i}, \varepsilon_{i}\right)=s\left(x_{i+1}, \varepsilon_{i+1}\right), i=1 \ldots n-1$, where

$$
s(x, \varepsilon)=\left\{\begin{array}{ll}
s x & \text { if } \varepsilon=+, \\
t x & \text { if } \varepsilon=-,
\end{array} \quad t(x, \varepsilon)= \begin{cases}t x & \text { if } \varepsilon=+, \\
s x & \text { if } \varepsilon=-.\end{cases}\right.
$$

The equivalence relation on words, and the composition, to obtain the free groupoid are 
defined in a manner analogous to the usual definition of free group, and the graph morphism $i: X \rightarrow F(X)$ sends an arrow $x$ to $[x]$, the equivalence class of the word $(x,+)$.

A groupoid $G$ is called connected if $G(a, b)$ is non-empty for all $a, b \in \operatorname{Ob}(G)$. The maximal connected subgroupoids of $G$ are called the (connected) components of $G$.

If $a$ is an object of the groupoid $G$, then the set $G(a, a)$ inherits a group structure from the composition on $G$; this is called the object group of $G$ at $a$ and is written also as $G(a)$. The groupoid $G$ is called simply connected if all its object groups are trivial. If it is connected and simply connected, it is called 1-connected, or a tree groupoid.

A standard example of a tree groupoid is the indiscrete, or square, groupoid $I(S)$ on a set $S$. This has object set $S$ and arrow set $S \times S$, with $s, t: S \times S \rightarrow S$ being the first and second projections. The composition on $I(S)$ is given by

$$
(a, b)(b, c)=(a, c), a, b, c \in S \text {. }
$$

A graph $X$ is called connected if the free groupoid $F(X)$ on $X$ is connected, and is called a forest if every object group $F(X)(a)$ of $F(X), a \in \mathrm{Ob}(X)$, is trivial. A connected forest is called a tree. If $X$ is a tree, then $F(X)$ is a tree groupoid.

\subsection{Retractions}

Let $G$ be a connected groupoid. Let $a_{0}$ be an object of $G$. For each object $a$ of $G$ choose an arrow $\tau a: a \rightarrow a_{0}$, with $\tau a_{0}=1_{a_{0}}$. Then an isomorphism

$$
\varphi: G \rightarrow G\left(a_{0}\right) \times I(\mathrm{Ob}(G))
$$

is given by $g \mapsto\left((\tau a)^{-1} g(\tau b),(a, b)\right), g \in G(a, b), a, b \in \mathrm{Ob}(G)$. The composition of $\varphi$ with the projection yields a morphism $\rho: G \rightarrow G\left(a_{0}\right)$ which we call a deformation retraction, since it is the identity on $G\left(a_{0}\right)$ and is in fact homotopic to the identity morphism of $G$, though we do not elaborate on this fact here.

It is also standard $[4,8.1 .5]$ that a connected groupoid $G$ is isomorphic to the free product groupoid $G\left(a_{0}\right) * T$ where $a_{0} \in \mathrm{Ob}(G)$ and $T$ is any wide, tree subgroupoid of $G$. The importance of this is as follows.

Suppose that $X$ is a graph which generates the connected groupoid $G$. Then $X$ is connected. Choose a maximal tree $T$ in $X$. Then $T$ determines for each $a_{0}$ in $\mathrm{Ob}(G)$ a retraction $\rho_{T}: G \rightarrow G\left(a_{0}\right)$ and the isomorphisms

$$
G \cong G\left(a_{0}\right) * I(\mathrm{Ob}(G)) \cong G\left(a_{0}\right) * F(T)
$$

show that a morphism $G \rightarrow K$ from $G$ to a groupoid $K$ is completely determined by a morphism of groupoids $G\left(a_{0}\right) \rightarrow K$ and a graph morphism $T \rightarrow K$ which agree on the object $a_{0}$.

We shall use later the following proposition, which is a special case of $[4,6.7 .3]$ :

Proposition 4.1. Let $G, H$ be groupoids with the same set of objects, and let $\varphi: G \rightarrow H$ be a morphism of groupoids which is the identity on objects. Suppose that $G$ is connected and $a_{0} \in \mathrm{Ob}(G)$. Choose a retraction $\rho: G \rightarrow G\left(a_{0}\right)$. Then there is a retraction $\sigma: H \rightarrow$ $H\left(a_{0}\right)$ such that the following diagram, where $\varphi^{\prime}$ is the restriction of $\varphi$,

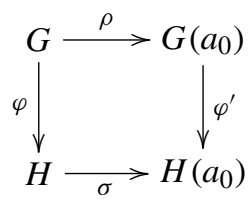


is commutative and is a pushout of groupoids.

\subsection{Normal subgroupoids and quotient groupoids}

Let $G$ be a groupoid. A subgroupoid $N$ of $G$ is called normal if $N$ is wide in $G$ (that is $\mathrm{Ob}(N)=\mathrm{Ob}(G))$ and for any objects $a, b$ of $G$ and $g$ in $G(b, a), g^{-1} N(b) g=N(a)$.

Let $\varphi: G \rightarrow H$ be a morphism of groupoids. Then $\operatorname{Ker} \varphi$, the wide subgroupoid of $G$ whose elements are all $g$ in $G$ such that $\varphi g$ is an identity of $H$, is a normal subgroupoid of $G$. If $\mathrm{Ob}(f)$ is injective then $\operatorname{Ker} \varphi$ is totally disconnected; that is, $(\operatorname{Ker} \varphi)(a, b)=\emptyset$ if $a \neq b$.

A morphism $\varphi: G \rightarrow H$ is said to annihilate a subgraph $X$ of $G$ if $\varphi(X)$ is a discrete subgroupoid of $H$. Thus $\operatorname{Ker} \varphi$ is the largest subgroupoid of $G$ annihilated by $\varphi$. The next proposition gives the existence of quotient groupoids.

Proposition 4.2. Let $N$ be a totally disconnected, normal subgroupoid of $G$. Then there is a groupoid $G / N$ and a morphism $p: G \rightarrow G / N$ such that $p$ annihilates $N$ and is universal for morphisms from $G$ which annihilate $N$.

Proof. We define $\mathrm{Ob}(G / N)=\mathrm{Ob}(G)$. If $a, b \in \mathrm{Ob}(G)$ we define $(G / N)(a, b)$ to consist of all cosets $N(a) g, g \in G(a, b)$. The multiplication of $G$ is inherited by $G / N$, which becomes a groupoid.

The morphism $p: G \rightarrow G / N$ is the identity on objects, and on elements is defined by $g \mapsto N(s g) g$. Clearly $p$ is a morphism and Ker $p=N$.

The remainder of the proof is clear.

We call $G / N$ a quotient groupoid of $G$.

\subsection{Presentations of groupoids}

We now consider relations in a groupoid. Suppose given for each object $a$ of the groupoid $G$ a set $R(a)$ of elements of $G(a)$; thus $R$ can be regarded as a wide, totally disconnected subgraph of $G$. The normal closure $N(R)$ of $R$ is the smallest wide normal subgroupoid of $G$ which contains $R$. This obviously exists since the intersection of any family of normal subgroupoids of $G$ is again a normal subgroupoid of $G$. Further, $N(R)$ is totally disconnected since the family of object groups of any normal subgroupoid $N$ of $G$ is again a normal subgroupoid of $G$.

Alternatively, $N=N(R)$ can be constructed explicitly. Let $a$ be an object of $G$. By a consequence of $R$ at $a$ is meant either the identity of $G$ at $a$, or any product

$$
\tau=g_{1}^{-1} r_{1}^{\varepsilon_{1}} g_{1} \ldots g_{n}^{-1} r_{n}^{\varepsilon_{n}} g_{n},
$$

in which $n \geqslant 1, g_{i} \in G\left(a_{i}, a\right)$ for some object $a_{i}$ of $G, \varepsilon_{i}= \pm 1$ and $r_{i}$ is an element of $R\left(a_{i}\right)$. Clearly, the set $N(a)$ of consequences of $R$ at $a$ is a subgroup of $G(a)$, and the family $N=(N(a): a \in \mathrm{Ob}(G))$ of these groups is a totally disconnected normal subgroupoid of $G$ containing $R$. Clearly $N=N(R)$.

The projection $p: G \rightarrow G / N(R)$ has the following universal property. If $f: G \rightarrow H$ is any morphism which annihilates $R$ then there is a unique morphism $f: G / N(R) \rightarrow H$ such that $f p=f$. We call $G / N(R)$ the groupoid $G$ with the relations $r=1, r \in R$.

In applications, we are often given $G, R$ as above, and wish to describe the object groups of $G / N(R)$. These are determined by the following result. 
Proposition 4.3. $[4,8.3 .3]$ Let $G$ be connected, let $a_{0} \in \mathrm{Ob}(G)$ and let $\rho: G \rightarrow G\left(a_{0}\right)$ be a deformation retraction. Let $H=G / N(R)$. Then $H\left(a_{0}\right)$ is isomorphic to the group $G\left(a_{0}\right)$ with the relations

$$
\rho(r)=1, r \in R
$$

Proof. The proof follows from Proposition 4.1, with $H=G / N$ and $\varphi=p: G \rightarrow G / N$ the quotient morphism. Details are given in [4].

\section{Crossed modules and free crossed modules over groupoids}

The theory of crossed modules and free crossed modules is due to Whitehead [40]. Expositions are given in, for example, $[\mathbf{1 7}, \mathbf{3 1}]$. In order to obtain an algebraic model of universal covers, we need the corresponding definitions for the groupoid case, due to Brown and Higgins in [11].

Let $\Phi$ be a groupoid. A crossed $\Phi$-module consists of:

(i) a totally disconnected groupoid $M$ with the same object set as $\Phi$;

(ii) a morphism $\mu: M \rightarrow \Phi$ of groupoids which is the identity on objects; and

(iii) a (partial) action of the groupoid $\Phi$ on the right of the groupoid $M$ via $\mu$.

This last condition means that if $x \in \Phi(a, b), m \in M(p)$, then $m^{x} \in M(b)$ and the usual laws of an action apply, namely $m^{1}=m,\left(m^{x}\right)^{y}=m^{x y},(m n)^{x}=m^{x} n^{x}$ whenever the terms are defined.

The axioms for a crossed module are:

(CM1) $\mu\left(m^{x}\right)=x^{-1}(\mu m) x$,

(CM2) $n^{-1} m n=m^{\mu n}$,

for all $m, n \in M, x \in \Phi$ and whenever the terms are defined.

Such a crossed $\Phi$-module is written $(M, \mu, \Phi)$ or $\mu: M \rightarrow \Phi$, or simply as $M$.

A morphism from a crossed module $\mu: M \rightarrow \Phi$ to a crossed module $v: N \rightarrow \Psi$ consists of a pair of morphisms of groupoids $f: \Phi \rightarrow \Psi, g: M \rightarrow N$ such that $\nu g=f \mu$ and $g\left(m^{x}\right)=(\mathrm{gm})^{f x}$ whenever $m^{x}$ is defined. This yields the category XMod of crossed modules and their morphisms.

There is also a category PXMod of precrossed modules, in which the axiom CM2) is dropped. The inclusion of categories XMod $\rightarrow$ PXMod has a left adjoint constructed as follows.

Let $\mu: M \rightarrow \Phi$ be a precrossed module. By a Peiffer element, or twisted commutator, is meant an element

$$
\langle m, n\rangle=m^{-1} n^{-1} m n^{\mu m}
$$

where $m, n \in M(p)$ for some object $p$. As in the group case (see [17, Proposition 2, p.158]) one proves that the Peiffer elements generate a normal $\Phi$-invariant subgroupoid $\langle M, M\rangle$ of $M$, and the quotient groupoid, $M^{a s s}=M /\langle M, M\rangle$, with the induced morphism $\mu^{\prime}: M^{\text {ass }} \rightarrow \Phi$, inherits the structure of the crossed module. This associated crossed module gives the reflection from the category PXMod of precrossed modules to the category XMod of crossed modules as required.

Let $\Phi$ be a groupoid, let $R$ be a totally disconnected graph with the same object set as $\Phi$, and let $\omega: R \rightarrow \Phi$ be a graph morphism which is the identity on objects. We define 
the free crossed module on $\omega$ to be a crossed module $\partial: C(\omega) \rightarrow \Phi$ together with a graph morphism $\bar{\omega}: R \rightarrow C(\omega)$ such that:

(i) $\partial \bar{\omega}=\omega$;

(ii) if $\mu: M \rightarrow \Phi$ is a crossed module and $g: R \rightarrow M$ is a graph morphism over the identity on objects such that $\mu g=\omega$, then there is a unique morphism $g^{\prime}: C(R) \rightarrow M$ of crossed $\Phi$-modules such that $g^{\prime} \bar{\omega}=g$.

Free crossed modules over groups were defined and constructed by Whitehead [40], and an exposition is given in [17]. The analogous construction for groupoids is as follows.

Let $\omega: R \rightarrow \Phi$ be given as above. One first forms the free groupoid $H(\omega)$ on the totally disconnected graph $Y$ with object set $\operatorname{Ob}(\Phi)$ where $Y(p)$ consists of pairs $(r, u)$ such that $r \in R(q), u \in \Phi(q, p)$. Let $\partial^{\prime}: H(\omega) \rightarrow \Phi$ be given by $(r, u) \mapsto u^{-1}(\omega r) u$, and let $\Phi$ operate on $H(\omega)$ by $(r, u)^{v}=(r, u v)$. This yields the free precrossed module on $\omega$, and the free crossed module is the associated crossed module $\partial: C(\omega)=H(\omega)^{\text {ass }} \rightarrow \Phi$.

Notice that the image $\partial(C(\omega))$ is the normal closure $N(\omega R)$ of $\omega R$ in $\Phi$.

It is useful to see this construction as a special case of the induced crossed modules of [10] (but for the groupoid case), which can be regarded as arising from a 'change of base' [6]. That is, $C(\omega)$ is isomorphic to the crossed module $\omega_{*} F(R)$ induced from the identity crossed module $1: F(R) \rightarrow F(R)$ by the morphism $\omega: F(R) \rightarrow \Phi$ determined by $\omega: R \rightarrow \Phi$. Further, we have a pushout of crossed modules

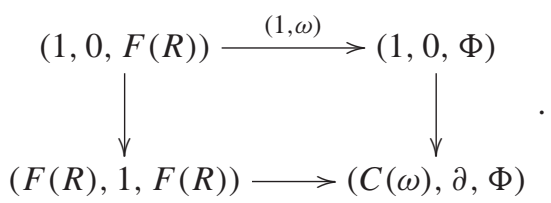

This allows a link with the two-dimensional Van Kampen Theorem of [10] (or rather, with the groupoid version formulated in all dimensions in [12]), to obtain a proof of a groupoid version of a well-known theorem of Whitehead [40], as follows.

Theorem 5.1. Let $U_{0}$ be a subset of the space $U$ and suppose the space $V$ is obtained from $U$ by attaching 2-cells by maps of pairs $f_{r}:\left(S^{1}, 1\right) \rightarrow\left(U, U_{0}\right), r \in R$. Then the family of second relative homotopy groups $\pi_{2}(V, U, p), p \in U_{0}$ form the free crossed module over the fundamental groupoid $\pi_{1}\left(U, U_{0}\right)$ on the graph morphism $\omega: R \rightarrow \pi_{1}\left(U, U_{0}\right)$ given by $\omega r=\left(f_{r}\right)_{*}(\iota)$, where $\iota$ here denotes a generator of the fundamental group $\pi_{1}\left(S^{1}, 1\right)$.

\section{Crossed complexes}

The basic geometric example of a crossed complex is the fundamental crossed complex $\pi X_{*}$ of a filtered space

$$
X_{*}: X_{0} \subseteq X_{1} \subseteq \cdots \subseteq X_{n} \subseteq \cdots \subseteq X
$$

Here $\pi_{1} X_{*}$ is the fundamental groupoid $\pi_{1}\left(X_{1}, X_{0}\right)$ and for $n \geqslant 2, \pi_{n} X_{*}$ is the family of relative homotopy groups $\pi_{n}\left(X_{n}, X_{n-1}, p\right)$ for all $p \in X_{0}$. These come equipped with the standard operations of $\pi_{1} X_{*}$ on $\pi_{n} X_{*}$ and boundary maps $\delta: \pi_{n} X_{*} \rightarrow \pi_{n-1} X_{*}$. The axioms for crossed complexes are those universally satisfied for this example.

The definition of a crossed complex generalises to the case of a set of base points definitions given by Blakers [2] (under the term 'group system') and Whitehead [40], under 
the term 'homotopy system' (except that he restricted it also to the free case). We recall this general definition from [11].

A crossed complex $C$ (over a groupoid) is a sequence of morphisms of groupoids over $C_{0}$

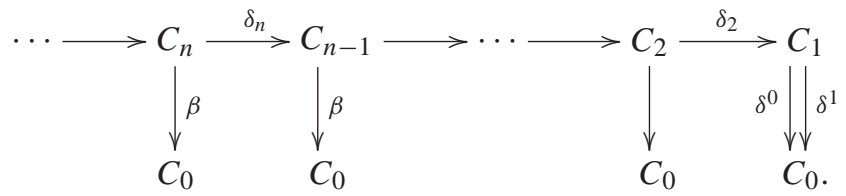

Here $\left\{C_{n}\right\}_{n} \geqslant 2$ is a family of groups with base point map $\beta$, and $\delta^{0}, \delta^{1}$ are the source and targets for the groupoid $C_{1}$. We further require given an operation of the groupoid $C_{1}$ on each family of groups $C_{n}$ for $n \geqslant 2$ such that:

(i) each $\delta_{n}$ is a morphism over the identity on $C_{0}$;

(ii) $C_{2} \rightarrow C_{1}$ is a crossed module over $C_{1}$;

(iii) $C_{n}$ is a $C_{1}$-module for $n \geqslant 3$;

(iv) $\delta: C_{n} \rightarrow C_{n-1}$ is an operator morphism for $n \geqslant 3$;

(v) $\delta \delta: C_{n} \rightarrow C_{n-2}$ is trivial for $n \geqslant 3$;

(vi) $\delta C_{2}$ acts trivially on $C_{n}$ for $n \geqslant 3$.

Because of axiom (iii) we shall write the composition in $C_{n}$ additively for $n \geqslant 3$, but we will use multiplicative notation in dimensions 1 and 2.

Let $C$ be a crossed complex. Its fundamental groupoid $\pi_{1} C$ is the quotient of the groupoid $C_{1}$ by the normal, totally disconnected subgroupoid $\delta C_{2}$. The rules for a crossed complex give $C_{n}$, for $n \geqslant 3$, the induced structure of a $\pi_{1} C$-module.

A morphism $f: C \rightarrow D$ of crossed complexes is a family of groupoid morphisms $f_{n}: C_{n} \rightarrow D_{n}(n \geqslant 0)$ which preserves all the structure. This defines the category Crs of crossed complexes. The fundamental groupoid now gives a functor $\pi_{1}$ : Crs $\rightarrow$ Gpd. This functor is left adjoint to the functor $i: \mathrm{Gpd} \rightarrow \mathrm{Crs}$, where for a groupoid $G$ the crossed complex $i G$ agrees with $G$ in dimensions 0 and 1 , and is otherwise trivial.

An $m$-truncated crossed complex $C$ consists of all the structure defined above, but only for $n \leqslant m$. In particular, an $m$-truncated crossed complex is for $m=0,1,2$ simply a set, a groupoid, and a crossed module respectively.

\section{Covering morphisms of groupoids and crossed complexes}

For the convenience of readers, and to fix the notation, we recall here the basic facts on covering morphisms of groupoids.

Let $G$ be a groupoid. For each object $a$ of $G$ the star of $a$ in $G$, denoted by $\operatorname{St}_{G} a$, is the union of the sets $G(a, b)$ for all objects $b$ of $G$, that is $\operatorname{St}_{G} a=\{g \in G: s g=a\}$. A morphism $p: \widetilde{G} \rightarrow G$ of groupoids is a covering morphism if for each object $\widetilde{a}$ of $\widetilde{G}$ the restriction of $p$

$$
\mathrm{St}_{\tilde{G}} \widetilde{a} \rightarrow \mathrm{St}_{G} p \widetilde{a}
$$

is bijective. In this case $\widetilde{G}$ is called a covering groupoid of $G$.

A basic result for covering groupoids is unique path lifting. That is, let $p: \widetilde{G} \rightarrow G$ be a covering morphism of groupoids, and let $\left(g_{1}, g_{2}, \ldots, g_{n}\right)$ be a sequence of composable 
elements of $G$. Let $\tilde{a} \in \mathrm{Ob}(\widetilde{G})$ be such that $p \tilde{a}$ is the starting point of $g_{1}$. Then there is a unique composable sequence $\left(\tilde{g}_{1}, \tilde{g}_{2}, \ldots, \tilde{g}_{n}\right)$ of elements of $\widetilde{G}$ such that $\tilde{g}_{1}$ starts at $\tilde{a}$ and $p \tilde{g}_{i}=g_{i}, i=1, \ldots, n$.

If $G$ is a groupoid, the category GpdCov / $G$ of coverings of $G$ has as objects the covering morphisms $p: H \rightarrow G$, and has as arrows (morphisms) the commutative diagrams of morphisms of groupoids, where $p$ and $q$ are covering morphisms,

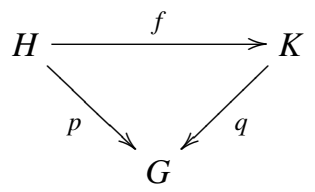

By a result of [4], $f$ also is a covering morphism. It is convenient to write such a diagram as a triple $(f, p, q)$. The composition in GpdCov/ $G$ is then given as usual by

$$
(g, q, r)(f, p, q)=(g f, p, r) .
$$

It is a standard result (see for example $[30,3]$ ) that the category GpdCov/ $G$ is equivalent to the functor category Set ${ }^{\text {op }}$. Thus if $X: G^{\text {op }} \rightarrow$ Set is a functor, then $\widetilde{G}=X \rtimes G$ has object set the disjoint union of the sets $X(a)$ for $a \in \mathrm{Ob}(G)$ and arrows $x \rightarrow y$ the pairs $(x, g)$ such that $x \in X(s g)$ and $X(g)(x)=y$. This 'semidirect product' or 'Grothendieck construction' is useful for constructing covering morphisms of the groupoid $G$. For example, if $a$ is an object of the transitive groupoid $G$, and $A$ is a subgroup of the object group $G(a)$, then the groupoid $G$ operates on the family of cosets $\left\{A h: h \in \mathrm{St}_{G} a\right\}$, by $(A h) . g=A h g$ whenever $h g$ is defined, and the associated covering morphism $\widetilde{G} \rightarrow G$ defines the cover $\widetilde{G}$ of the groupoid $G$ determined by the subgroup $A$. When $A$ is trivial, this gives the universal cover at $a$ of the groupoid $G$. In particular, this gives the universal covering groupoid of a group.

We now give the generalisation of this notion to crossed complexes.

Definition 7.1. [32] A morphism $p: \widetilde{C} \rightarrow C$ of crossed complexes is a covering morphism if

(i) the morphism $p_{1}:\left(\widetilde{C}_{1}, \widetilde{C}_{0}\right) \rightarrow\left(C_{1}, C_{0}\right)$ is a covering morphism of groupoids;

(ii) for each $n \geqslant 2$ and $\widetilde{x} \in \widetilde{C}_{0}$, the morphism of groups $p_{n}: \widetilde{C}_{n}(\widetilde{x}) \rightarrow C_{n}(p \tilde{x})$ is an isomorphism.

In such cases we call $\widetilde{C}$ a covering crossed complex of $C$.

This definition may also be expressed in terms of the unique covering homotopy property. For more details (but there with emphasis on fibrations) see [16].

Proposition 7.2. Let $p: \widetilde{C} \rightarrow C$ be a covering morphism of crossed complexes and let $\tilde{a} \in \mathrm{Ob}(\widetilde{C})$. Let $a=p \tilde{a}$, and let $K=p_{0}^{-1}(a) \subseteq \mathrm{Ob}(\widetilde{C})$. Then $p$ induces isomorphisms $\pi_{n}(\widetilde{C}, \tilde{a}) \rightarrow \pi_{n}(C, a)$ for $n \geqslant 2$ and a sequence

$$
1 \rightarrow \pi_{1}(\widetilde{C}, \tilde{a}) \rightarrow \pi_{1}(C, a) \rightarrow K \rightarrow \pi_{0}(\widetilde{C}) \rightarrow \pi_{0}(C)
$$

which is exact in the sense of the exact sequence of a fibration of groupoids.

The comment about exactness has to do with operations on the pointed sets; see $[3,4]$. The proof of the proposition is easy, and is omitted here.

The following result gives a basic geometric example of a covering morphism of crossed complexes. 
Theorem 7.3. Let $X_{*}$ and $Y_{*}$ be filtered spaces and let $f: X \rightarrow Y$ be a covering map of spaces such that for each $n \geqslant 0, f_{n}: X_{n} \rightarrow Y_{n}$ is also a covering map with $X_{n}=f^{-1}\left(Y_{n}\right)$. Then $\pi f: \pi X_{*} \rightarrow \pi Y_{*}$ is a covering morphism of crossed complexes.

Proof. By a result of [4], $\pi f: \pi_{1} X_{1} \rightarrow \pi_{1} Y_{1}$ is a covering morphism of groupoids. Since $X_{0}=f^{-1}\left(Y_{0}\right)$, the restriction of $\pi_{1} f$ to $\pi_{1}\left(X_{1}, X_{0}\right) \rightarrow \pi_{1}\left(Y_{1}, Y_{0}\right)$ is also a covering morphism of groupoids. Now for each $n \geqslant 2$ and for each $x_{0} \in X_{0}, f_{*}: \pi_{n}\left(X_{n}, X_{n-1}, x_{0}\right) \rightarrow$ $\pi_{n}\left(Y_{n}, Y_{n-1}, p\left(x_{0}\right)\right)$ is an isomorphism (see for example, [33]).

Here is an important method of constructing new covering morphisms.

Proposition 7.4. Let $p: \widetilde{C} \rightarrow C$ be a covering morphism of crossed complexes. Then the induced morphism $\pi_{1}(p): \pi_{1} \widetilde{C} \rightarrow \pi_{1} C$ is a covering morphism of groupoids.

Proof. Let $\tilde{x} \in \widetilde{C}_{0}$. We will show that $p_{\tilde{x}}^{\prime}: \mathrm{St}_{\pi_{1}} \widetilde{C} \tilde{x} \rightarrow \mathrm{St}_{\pi_{1} C} p \tilde{x}$ is bijective. Let $[a] \in$ $\mathrm{St}_{\pi_{1} C} p \tilde{x}$, where $a \in \mathrm{St}_{C} p \tilde{x}$. Since $p$ is a covering morphism, there exists a unique $\tilde{a}$ of $\mathrm{St}_{\tilde{C}} \tilde{x}$ such that $p \tilde{a}=a$. So $p_{\tilde{x}}^{\prime}[\tilde{a}]=[a]$ and thus $p_{\tilde{x}}^{\prime}$ is surjective.

Now suppose that $p_{\tilde{x}}^{\prime}[\tilde{a}]=p_{\tilde{x}}^{\prime}[\tilde{b}]$. Then $(p \tilde{b})^{-1} p \tilde{a} \in \delta C_{2}(p \tilde{x})$, which implies that $(p \tilde{b})^{-1}(p \tilde{a})=\delta p \tilde{c}$ for a unique $\tilde{c} \in \widetilde{C}_{2}(\tilde{x})$. Because $p$ is a covering morphism, we need only show that $(\tilde{b})^{-1} \tilde{a}=\delta \tilde{c}$. This follows by star injectivity. Therefore $p_{\tilde{x}}^{\prime}$ is injective, and so is bijective. Hence $\pi_{1}(p)$ is a covering morphism of groupoids.

Let $C$ be a crossed complex. We write $\operatorname{CrsCov} / C$ for the full subcategory of the slice category $\mathrm{Crs} / C$ whose objects are the covering morphisms of $C$.

Proposition 7.5. Suppose given a pullback diagram of crossed complexes

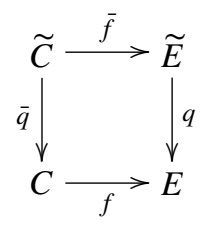

in which $q$ is a covering morphism. Then $\bar{q}$ is a covering morphism.

We omit the proof. The groupoid case is done in [4, 9.7.6]. See also [9] for uses of pullbacks of covering morphisms of groupoids.

Our next result is the analogue for covering morphisms of crossed complexes of a classical result for covering maps of spaces [4, 9.6.1].

Theorem 7.6. If $C$ is a crossed complex, then the functor $\pi_{1}:$ Crs $\rightarrow$ Gpd induces an equivalence of categories

$$
\pi_{1}^{\prime}: \operatorname{CrsCov} / C \rightarrow \operatorname{GpdCov} /\left(\pi_{1} C\right) .
$$

Proof. If $p: \widetilde{C} \rightarrow C$ is a covering morphism of crossed complexes, then $\pi_{1} p: \pi_{1} \widetilde{C} \rightarrow$ $\pi_{1} C$ is a covering morphism of groupoids, by Proposition 7.4. Since $\pi_{1}$ is a functor, we also obtain the functor $\pi_{1}^{\prime}$. To prove $\pi_{1}^{\prime}$ is an equivalence of categories, we construct a functor $\rho: \operatorname{GpdCov} /\left(\pi_{1} C\right) \rightarrow \operatorname{CrsCov} / C$, and prove that there are equivalences of functors $1 \simeq \rho \pi_{1}^{\prime}$ and $1 \simeq \pi_{1}^{\prime} \rho$. 
Let $C$ be a crossed complex, and let $q: D \rightarrow \pi_{1} C$ be a covering morphism of groupoids. Let $\widetilde{C}$ be given by the pullback diagram in the category of crossed complexes:

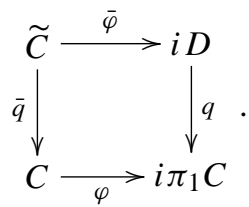

By Proposition 7.5, $\bar{q}: \widetilde{C} \rightarrow C$ is a covering morphism of crossed complexes.

We define the functor $\rho$ by $\rho(q)=\bar{q}$, and extend $\rho$ in the obvious way to morphisms.

The natural transformation $\pi_{1}^{\prime} \rho \simeq 1$ is defined on a covering morphism $q: D \rightarrow \pi_{1} C$ to be the composite morphism

$$
\lambda: \pi_{1}(\widetilde{C}) \stackrel{\pi_{1}(\bar{\varphi})}{\longrightarrow} \pi_{1}(i D) \cong D
$$

where $\bar{\varphi}: \widetilde{C} \rightarrow i D$ is given in diagram (11). The proof that $\lambda$ is an isomorphism is simple, and is left to the reader.

To prove that $1 \simeq \rho \pi_{1}^{\prime}$, we show that the following diagram is a pullback:

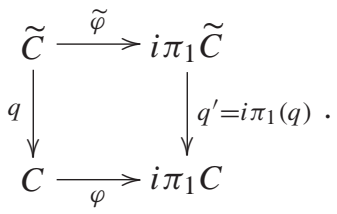

This is clear in dimension 0 and in dimensions $\geqslant 2$. For the case of dimension 1 , let $c: x \rightarrow y$ in $C$, and $[\tilde{c}] \in\left(\pi_{1} \widetilde{C}\right)(\tilde{x}, \tilde{y})$ be such that $q[\tilde{c}]=\varphi(c)$. Then there exists a unique $\tilde{c}^{\prime}: \tilde{x} \rightarrow \tilde{y}$ such that $\widetilde{\varphi}\left(\tilde{c}^{\prime}\right)=[\tilde{c}]$ and $\bar{q}\left(\tilde{c}^{\prime}\right)=c$. Now, $\bar{q}\left(\tilde{c} \delta \widetilde{C}_{2}(\tilde{x})\right)=\varphi(c)=c \delta C_{2}(x)$. This implies that $(\bar{q} \tilde{c}) \delta C_{2}(x)=c \delta C_{2}(x)$. So $\bar{q}(\tilde{c})=c\left(\delta c_{2}\right)$ for some $c_{2} \in C_{2}(x)$. Therefore there exists a unique $\tilde{c}_{2} \in \widetilde{C}_{2}(\tilde{x})$ covering $c_{2}$, and $\bar{q}\left(\tilde{c}\left(\delta \tilde{c}_{2}\right)^{-1}\right)=c$. So the above diagram is a pullback, and thus we have proved that $1 \simeq \rho \pi_{1}^{\prime}$. This proves the equivalence of the two categories.

\section{Covering morphisms and colimits}

In this section we give a result due to Howie [32, Theorem 5.1], which we use to prove that covering crossed complexes of free crossed complexes are free.

Theorem 8.1. Let $p: A \rightarrow B$ be a morphism of crossed complexes. Then $p$ is a fibration if and only if the pullback functor $p^{*}: \mathrm{Crs} / B \rightarrow \mathrm{Crs} / A$ has a right adjoint.

As a consequence we get the following corollary.

Corollary 8.2. If $p: A \rightarrow B$ is a covering morphism of crossed complexes, then $p^{*}$ : $\mathrm{Crs} / B \rightarrow \mathrm{Crs} / A$ preserves all colimits.

\section{Coverings of free crossed complexes}

We recall here a definition from [13]. A free basis for a crossed complex $C$ consists of subgraphs $X_{n}$ of $C_{n}$ for all $n \geqslant 1$ such that $C_{1}$ is the free groupoid on $X_{1}, C_{2}$ is the 
free crossed $C_{1}$-module on the restriction $\delta_{2}^{\prime}: X_{2} \rightarrow C_{1}$, and for $n \geqslant 3, C_{n}$ is the free $\pi_{1} C$-module on $X_{n}$.

Following [16] we write $\mathbb{C}(n)$ for the crossed complex freely generated by one generator $c_{n}$ in dimension $n$. So $\mathbb{C}(0)$ is the singleton set $\{1\} ; \mathbb{C}(1)$ is the groupoid $\ell$ which has two objects 0,1 and non-identity elements $c_{1}: 0 \rightarrow 1$ and $c_{1}^{-1}: 1 \rightarrow 0$; and for $n \geqslant 2, \mathbb{C}(n)$ is in dimensions $n$ and $n-1$ an infinite cyclic group with generators $c_{n}$ and $\delta c_{n}$ respectively, and is otherwise trivial. Thus if $C$ is a crossed complex, then an element $c \in C_{n}$ is completely specified by a morphism $\hat{c}: \mathbb{C}(n) \rightarrow C$ such that $\hat{c}\left(c_{n}\right)=c$, and $\delta(c)=\hat{c}\left(\delta c_{n}\right)$.

Let $\mathbb{S}(n-1)$ be the subcomplex of $\mathbb{C}(n)$ which agrees with $\mathbb{C}(n)$ up to dimension $n-1$, and is trivial otherwise. If $\mathbf{E}^{n}$ and $\mathbf{S}^{n-1}$ denote the skeletal filtrations of the standard $n$-ball and $(n-1)$-sphere, where $E^{0}=\{0\}, S^{-1}=\varnothing, E^{1}=I=\{0,1\} \cup e^{1}, S^{0}=\{0,1\}$, and for $n \geqslant 2, E^{n}=\{1\} \cup e^{n-1} \cup e^{n}, S^{n-1}=\{1\} \cup e^{n-1}$, then it is clear that for all $n \geqslant 0, \mathbb{C}(n) \cong \pi \mathbf{E}^{n}$ and $\mathbb{S}(n-1) \cong \pi \mathbf{S}^{n-1}$.

We now model for crossed complexes the process for spaces known as attaching cells. Let $A$ be any crossed complex. A sequence of morphisms $j_{n}: C^{n-1} \rightarrow C^{n}$ may be defined with $C^{0}=A$ by choosing any family of morphisms $\mathbb{S}\left(m_{\lambda}-1\right) \rightarrow C^{n-1}$ for any $\lambda \in \Lambda_{n}$ and any $m_{\lambda}$, and forming the pushout

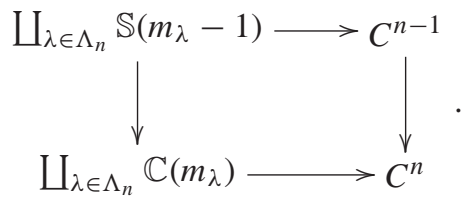

Let $C=\operatorname{colim}_{n} C^{n}$, and let $j: A \rightarrow C$ be the canonical morphism. The morphism $j: A \rightarrow C$ is called a relatively free crossed complex morphism. If $A$ is empty, then we call $C$ a free crossed complex.

The importance of the definition is as follows:

Proposition 9.1. If $C$ is a free crossed complex on $X_{*}$, then a morphism $f: C \rightarrow D$ can be constructed inductively, provided that one is given the values $f_{n} x \in D_{n}, x \in X_{n}, n \geqslant 0$, and provided that the following geometric conditions are satisfied: (i) $\delta^{\alpha} f_{1} x=f_{0} \delta^{\alpha} x, x \in$ $X_{1}, \alpha=0,1$; (ii) $\beta f_{n}(x)=f_{0}(\beta x), x \in X_{n}, n \geqslant 2$; (iii) $\delta_{n} f_{n}(x)=f_{n-1} \delta_{n}(x), x \in$ $X_{n}, n \geqslant 2$.

Notice that in (iii), $f_{n-1}$ has to be defined on all of $C_{n-1}$ before this condition can be verified.

We now show that freeness can be lifted to covering crossed complexes.

Theorem 9.2. Suppose given a pullback square of crossed complexes

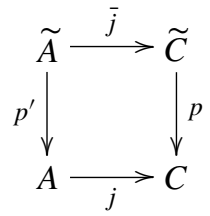

in which $p$ is a covering morphism and $j: A \rightarrow C$ is relatively free. Then $\bar{j}: \widetilde{A} \rightarrow \widetilde{C}$ is relatively free. 
Proof. We suppose given the sequence of diagrams 12. Let $\hat{C}^{n}=p^{-1}\left(C^{n}\right)$. By Corollary 8.2, the following diagram is a pushout:

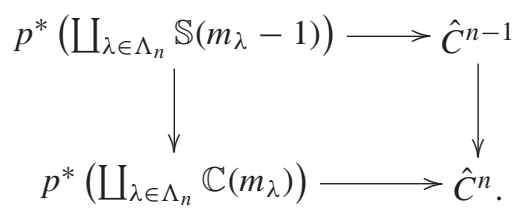

Since $p$ is a covering morphism, we can write $p^{*}\left(\bigsqcup_{\lambda \in \Lambda_{n}} \mathbb{C}\left(m_{\lambda}\right)\right)$ as $\bigsqcup_{\lambda \in \tilde{\Lambda}_{n}} \mathbb{C}\left(m_{\lambda}\right)$ for a suitable $\widetilde{\Lambda}_{n}$. This completes the proof.

Corollary 9.3. Let $p: \widetilde{C} \rightarrow C$ be a covering morphism of crossed complexes. If $C$ is free on $X_{*}$, then $\widetilde{C}$ is free on $p^{-1}\left(X_{*}\right)$.

A similar result to Corollary 9.3 applies in the $m$-truncated case.

The significance of these results is as follows. We start with an $m$-truncated free crossed resolution $C$ of a group $G$, so that we are given $\varphi: C_{1} \rightarrow G$, and $C$ is free on $X_{*}$, where $X_{n}$ is defined only for $n \leqslant m$. Our extension process of Section 10 will start by constructing the universal cover $p: \widetilde{C} \rightarrow C$ of $C$; this is the covering crossed complex corresponding to the universal covering groupoid $p_{0}: \widetilde{G} \rightarrow G$. By the results above, $\widetilde{C}$ is the free crossed complex on $p^{-1}\left(X_{*}\right)$. It also follows from Proposition 7.2 that the induced morphism $\widetilde{\varphi}: \widetilde{C} \rightarrow \widetilde{G}$ makes $\widetilde{C}$ a free crossed resolution of the contractible groupoid $\widetilde{G}$. Hence $\widetilde{C}$ is an acyclic and hence, since it is free, also a contractible crossed complex.

We now see the general context for the diagram (1) of Section 2 and the exposition there.

\section{Homotopies}

We follow the conventions for homotopies in [14]. Thus a homotopy $f^{0} \simeq f$ of morphisms $f^{0}, f: C \rightarrow D$ of crossed complexes is a pair $(h, f)$ where $h$ is a family of functions $h_{n}: C_{n} \rightarrow D_{n+1}$ with the following properties, in which $\beta c$ for $c \in C$ is $c$, if $c \in C_{0}$, is $\delta^{1} c$, if $c \in C_{1}$, and is $x$ if $c \in C_{n}(x), n \geqslant 2$. So we require [14, (3.1)]:

$$
\begin{aligned}
\beta h_{n}(c) & =\beta f(c) & & \text { for all } c \in C ; \\
h_{1}\left(c c^{\prime}\right) & =h_{1}(c)^{f c^{\prime}} h_{1}\left(c^{\prime}\right) & & \text { if } c, c^{\prime} \in C_{1} \text { and } c c^{\prime} \text { is defined; } \\
h_{2}\left(c c^{\prime}\right) & =h_{2}(c)+h_{2}\left(c^{\prime}\right) & & \text { if } c, c^{\prime} \in C_{2} \text { and } c c^{\prime} \text { is defined; } \\
h_{n}\left(c+c^{\prime}\right) & =h_{n}(c)+h_{n}\left(c^{\prime}\right) & & \text { if } c, c^{\prime} \in C_{n}, n \geqslant 3 \text { and } c+c^{\prime} \text { is defined; } \\
h_{n}\left(c^{c_{1}}\right) & =\left(h_{n} c\right)^{f c_{1}} & & \text { if } c \in C_{n}, n \geqslant 2, c_{1} \in C_{1}, \text { and } c^{c_{1}} \text { is defined. }
\end{aligned}
$$

Then $f^{0}, f$ are related by $[\mathbf{1 4},(3.14)]$

$$
f^{0}(c)= \begin{cases}\delta^{0} h_{0} c & \text { if } c \in C_{0}, \\ \left(h_{0} \delta^{0} c\right)(f c)\left(\delta_{2} h_{1} c\right)\left(h_{0} \delta^{1} c\right)^{-1} & \text { if } c \in C_{1}, \\ \left\{(f c)\left(h_{1} \delta_{2} c\right)\left(\delta_{3} h_{2} c\right)\right\}^{\left(h_{0} \beta c\right)^{-1}} & \text { if } c \in C_{2}, \\ \left\{f c+h_{n-1} \delta_{n} c+\delta_{n+1} h_{n} c\right\}^{\left(h_{0} \beta c\right)^{-1}} & \text { if } c \in C_{n}, n \geqslant 3 .\end{cases}
$$

The following is important for our computations. We saw in Proposition 9.1 that a morphism is specified by its values on a graded set of free generators. We now show that the same is true for homotopies. 
Proposition 10.1. If $C$ is a free crossed complex on a generating family $X_{n}, n \geqslant 0$, then a homotopy $(h, f): f^{0} \simeq f: C \rightarrow D$ is specified by the values $f x \in D_{n}, h x \in D_{n+1}, x \in$ $X_{n}, n \geqslant 0$ provided only that the following geometric conditions hold:

$$
\begin{aligned}
\delta^{0} f x & =f \delta^{0} x, \delta^{1} f x=f \delta^{1} x, x \in X_{1}, \delta f x=f \delta x, x \in X_{n}, n \geqslant 2, \\
\beta f x & =f \beta x, x \in X_{n}, n \geqslant 1, \beta h x=\beta f x, x \in X_{n}, n \geqslant 0 .
\end{aligned}
$$

Proof. All but the last condition are those given for the construction of $f$ in Proposition 9.1. The final fact we need is that for $n \geqslant 2$ the $f_{1}$-morphism $h_{n}$ is defined by its values on the generators in $X_{n}$, and this is standard.

This result is another aspect of the facts that (i) a homotopy $C \rightarrow D$ can also be regarded as a morphism $\mathbb{C}(1) \otimes C \rightarrow D$, where the tensor product is defined in [14], and (ii) the tensor product of free crossed complexes is free, as proved in [16].

From this we can deduce formulae for a retraction. Suppose, then, that in the above formulae we take $C=D, f^{0}=1_{C}, f=0$ where 0 denotes the constant morphism on $C$ mapping everything to a base point 0 . Then the homotopy $h: 1 \simeq 0$ must satisfy

$$
\begin{aligned}
\beta h_{n} c & =0 & & \text { if } c \in C, \\
\delta^{0} h_{0} c & =c & & \text { if } c \in C_{0}, \\
\delta_{2} h_{1} c & =\left(h_{0} \delta^{0} c\right)^{-1} c\left(h_{0} \delta^{1} c\right) & & \text { if } c \in C_{1}, \\
\delta_{3} h_{2} c & =\left(h_{1} \delta_{2} c\right)^{-1} c^{h_{0} \beta c} & & \text { if } c \in C_{2}, \\
\delta_{n+1} h_{n} c & =-h_{n-1} \delta_{n} c+c^{h_{0} \beta c} & & \text { if } c \in C_{n}, n \geqslant 3, \\
h_{n}\left(c^{c_{1}}\right) & =\left(h_{n} c\right) & & \text { if } c \in C_{n}, n \geqslant 2, c_{1} \in C_{1}, \text { and } c^{c_{1}} \text { is defined. }
\end{aligned}
$$

Further, in this case $h_{1}$ is a morphism by (14), and for $n \geqslant 2, h_{n}$ is by (16) a morphism, which by (17) trivialises the operations of $C_{1}$. All these conditions are necessary and sufficient for $h$ to be a contracting homotopy.

An $m$-truncated crossed complex $C$ is a crossed complex as earlier, except that $C_{n}$ and $\delta_{n}$ are defined only for $n \leqslant m$. Similarly, for a contracting homotopy $h$ of an $m$-truncated crossed complex $C$, we have $h_{n}$ defined only for $n<m$, and the above conditions hold where they make sense.

Our main result is now rather formal and straightforward to prove. It is to extend the pair $(C, h)$ of a partial free crossed resolution $C$ and partial contracting homotopy $h$ of the universal cover of $C$ by one step. Hence the process can be continued indefinitely.

Theorem 10.2. Let $m \geqslant 1$ and let $C$ be an $m$-truncated free crossed resolution of a group $G$. Let $p: \widetilde{C} \rightarrow C$ be the universal cover of $C$ so that $\widetilde{C}$ is an $m$-truncated free crossed resolution of the universal covering groupoid $p_{0}: \widetilde{G} \rightarrow G$ of $G$. Let $h$ be a partial contracting homotopy of $\widetilde{C}$. Suppose also that $\widetilde{C}_{m}$ is free on $\widetilde{X}_{m}$.

Let $\widetilde{X}_{m} \rightarrow X_{m+1}, \tilde{x} \mapsto x$, be a bijection to a set $X_{m+1}$ disjoint from $\widetilde{X}_{m}$. Define an extension e $(C)$ of $C$ to an $(m+1)$-truncated free crossed complex as follows.

For $m=1$, let $C_{2}=e(C)_{2}$ be the free crossed $C_{1}$-module on $X_{2}$ with $\delta_{2}: X_{2} \rightarrow C_{1}$ given by

$$
\delta_{2} x=p_{1}\left(\left(h_{0} \tilde{\delta}^{0} \tilde{x}\right)^{-1} \tilde{x}\left(h_{0} \tilde{\delta}^{1} \tilde{x}\right)\right), \tilde{x} \in \tilde{X}_{1}
$$


For $m=2$ let $C_{3}=e(C)_{3}$ be the free $G$-module on $X_{3}$ with $\delta_{3}: e(C)_{3} \rightarrow C_{2}$ defined by

$$
\delta_{3} x=p_{2}\left(\left(h_{1} \widetilde{\delta}_{2} \tilde{x}\right)^{-1} \tilde{x}^{h_{0} \beta \tilde{x}}\right), \quad \tilde{x} \in \widetilde{X}_{2} .
$$

For $m \geqslant 3$ let $C_{m+1}=e(C)_{m+1}$ be the free $G$-module on $X_{m+1}$ with $\delta_{m+1}: C_{m+1} \rightarrow C_{m}$ defined by

$$
\delta_{m+1} x=p_{m}\left(-h_{m-1} \tilde{\delta}_{m} \tilde{x}+\tilde{x}^{h_{0} \beta \tilde{x}}\right), \tilde{x} \in \tilde{X}_{m}
$$

Let $e(p): e(\widetilde{C}) \rightarrow e(C)$ be the induced covering morphism, extending $p$ by $p_{m+1}$ : $\widetilde{C}_{m+1} \rightarrow C_{m+1}$.

Define $h_{m}: \widetilde{C}_{m} \rightarrow \widetilde{C}_{m+1}$ on the basis $\widetilde{X}_{m}$ by $h_{m}(\tilde{x})=(1, x)$. Then this extension $e(h)$ of $h$ is a contracting homotopy of $e(\widetilde{C})$. Hence $e(C)$ is an $(m+1)$-truncated free crossed resolution of $G$.

If further there is a subset $Y$ of $X_{m+1}$ such that $\delta_{m+1} Y$ also generates Ker $\delta_{m}$, and a retraction $\xi: C_{m+1} \rightarrow C_{m+1}(Y)$ is given such that $\delta_{m+1} \xi(x)=\delta_{m+1}(x)$ for all $x \in X_{m+1}$, and $\xi$ is a $G$-morphism for $m \geqslant 2$, and a crossed $C_{1}$-morphism for $m=1$, then we may replace $C_{m+1}$ by $C_{m+1}(Y)$ and $h_{m}$ by $\xi h_{m}$ to again get an extension of the pair $(C, h)$ by one step.

Proof. The fact that we have a contracting homotopy is immediate from the definitions. It follows that $e(\widetilde{C})$ is exact, and so $e(C)$ is aspherical with $\pi_{1}(e(C))=G$.

Corollary 10.3. Under the assumptions of Theorem 10.2, if $m=1$ then $\operatorname{Ker} \varphi: C_{1} \rightarrow G$ is generated as a normal subgroup of $C_{1}$ by the elements:

$$
p_{1}\left(\left(h_{0} \tilde{\delta}^{0} \tilde{x}\right)^{-1} \tilde{x}\left(h_{0} \tilde{\delta}^{1} \tilde{x}\right)\right), \tilde{x} \in \widetilde{X}_{1} .
$$

For $m \geqslant 2$, Ker $\left(\delta_{m}: C_{m} \rightarrow C_{m-1}\right)$ is generated as a $G$-module by the elements:

$$
\begin{aligned}
& p_{2}\left(\left(h_{1} \tilde{\delta}_{2} \tilde{x}\right)^{-1} \tilde{x}^{h_{0} \beta \tilde{x}}\right), \quad \tilde{x} \in \widetilde{X}_{2}, \quad \text { if } m=2, \\
& p_{m}\left(-h_{m-1} \tilde{\delta}_{m} \tilde{x}+\tilde{x}^{h_{0} \beta \tilde{x}}\right), \quad \tilde{x} \in \widetilde{X}_{m}, \quad \text { if } m \geqslant 3 .
\end{aligned}
$$

We have now finally justified the process set out in Section 2 and illustrated with an example in Section 3.

Implementations of these procedures will be given in papers by other authors $[27,28]$.

\section{Examples}

\subsection{The standard crossed resolution of a group}

The standard crossed resolution of a group was defined by Huebschmann in [34] and applied also in, for example, $[\mathbf{1 8}, \mathbf{3 9}]$. Here we show how this resolution, with some differing conventions, arises from our procedure.

We start with a group $G$ and let $C_{1}=F(G)$, the free group on the set $G$, with generators written $[a], a \in G$. Let $\varphi: C_{1} \rightarrow G$ be the canonical morphism. This has a section $\sigma: G \rightarrow F(G), a \mapsto[a], a \neq 1,1 \mapsto 1$. This defines $h_{0}: G \rightarrow \widetilde{C}_{1}, a \mapsto\left(a,[a]^{-1}\right)$.

The Cayley graph of this presentation has arrows $(a,[b]): a \rightarrow a b$ so that

$$
h_{0}(a)^{-1}(a,[b]) h_{0}(a b)=\left(1,[a][b][a b]^{-1}\right) .
$$


So we may take $C_{2}$ to be the free crossed $C_{1}$-module on elements $[a, b]$ and define $\delta_{2}$ : $C_{2} \rightarrow C_{1}$ by

$$
\delta_{2}[a, b]=[a][b][a b]^{-1} .
$$

Then in the universal cover we can define $h_{1}: \widetilde{C}_{1} \rightarrow \widetilde{C}_{2}(1)$ by $(a,[b]) \mapsto(1,[a, b])$.

Theorem 11.1. There is a free crossed $C_{*}(G)$ resolution of a group $G$ in which $C_{n}(G)$ $i$ free on the set $G^{n}$ with generators written $\left[a_{1}, a_{2}, \ldots, a_{n}\right], a_{i} \in G$, with contracting homotopy of the universal cover given by $\left(a,\left[a_{1}, a_{2}, \ldots, a_{n}\right]\right) \mapsto\left(1,\left[a, a_{1}, a_{2}, \ldots, a_{n}\right]\right)$, and boundary $\delta_{n}: C_{n}(G) \rightarrow C_{n-1}(G)$ given by $\delta_{2}$ as above,

$$
\delta_{3}[a, b, c]=[a, b c][a b, c]^{-1}[a, b]^{-1}[b, c]^{[a]^{-1}},
$$

and for $n \geqslant 4$

$$
\begin{array}{r}
\delta_{n}\left[a_{1}, a_{2}, \ldots, a_{n}\right]=\left[a_{2}, \ldots, a_{n}\right]^{a_{1}^{-1}}+\sum_{i=1}^{n-1}(-1)^{i}\left[a_{1}, a_{2}, \ldots, a_{i-1}, a_{i} a_{i+1}, a_{i+2}, \ldots, a_{n}\right] \\
+(-1)^{n}\left[a_{1}, a_{2}, \ldots, a_{n-1}\right] .
\end{array}
$$

Proof. We first verify

$$
\begin{aligned}
\tilde{\delta}_{3} h_{2}(a,[b, c]) & =h_{1} \widetilde{\delta}_{2}(a,[b, c])^{-1}(a,[b, c])^{\left(a,[a]^{-1}\right)} \\
& =h_{1}\left(1, a,[b][c][b c]^{-1}\right)^{-1}\left(1,[b, c]^{[a]^{-1}}\right) \\
& =h_{1}\left((a,[b])(a b,[c])\left(a b c,[b c]^{-1}\right)\right)^{-1}\left(1,[b, c]^{[a]^{-1}}\right) \\
& =h_{1}\left((a,[b])(a b,[c])(a,[b c])^{-1}\right)^{-1}\left(1,[b, c]^{[a]^{-1}}\right) \\
& =\left(1,[a, b c][a b, c]^{-1}[a, b]^{-1}[b, c]^{[a]^{-1}}\right) .
\end{aligned}
$$

In order to have a contracting homotopy we require for $n \geqslant 3$

$$
\begin{aligned}
& \tilde{\delta}_{n+1} h_{n}\left(a_{1},\left[a_{2}, \ldots, a_{n+1}\right]\right) \\
& =-h_{n-1} \widetilde{\delta}_{n}\left(a_{1},\left[a_{2}, \ldots, a_{n+1}\right]\right)+\left(1,\left[a_{2}, \ldots, a_{n+1}\right]^{a_{1}^{-1}}\right) \\
& =\left(1,\left[a_{2}, \ldots, a_{n+1}\right]^{a_{1}^{-1}}+\sum_{i=1}^{n}(-1)^{i}\left[a_{1}, \ldots, a_{i} a_{i+1}, \ldots, a_{n+1}\right]\right. \\
& \left.\quad+(-1)^{n+1}\left[a_{1}, a_{2}, \ldots, a_{n}\right]\right) .
\end{aligned}
$$

This completes the proof that the family $h_{n}$ give a contracting homotopy, and thus that $C_{*}(G)$ is a resolution.

Remark 11.2. The formulae for the differential given above are different in detail from those given in $[34,13,39]$. This reflects only the different conventions used.

\subsection{A small crossed resolution of finite cyclic groups}

We would like to thank A. Heyworth for discussions on this section.

We write $\mathrm{C}$ for the (multiplicative) infinite cyclic group with generator $x$, and $\mathrm{C}_{r}$ for the finite cyclic group of order $r$ with generator $t$. Let $\varphi: \mathrm{C} \rightarrow \mathrm{C}_{r}$ be the morphism sending 
$x$ to $t$. We show how the inductive procedure given earlier recovers the small free crossed resolution of $C_{r}$ given in [19], together with a contracting homotopy of the universal cover.

Let $p_{0}: \widetilde{\mathrm{C}}_{r} \rightarrow \mathrm{C}_{r}$ be the universal covering morphism, and let $p_{1}: \widetilde{\mathrm{C}} \rightarrow \mathrm{C}$ be the induced cover of $\mathrm{C}$. Then $\widetilde{\mathrm{C}}$ is the free groupoid on the Cayley graph $\widetilde{X}$ pictured as follows:

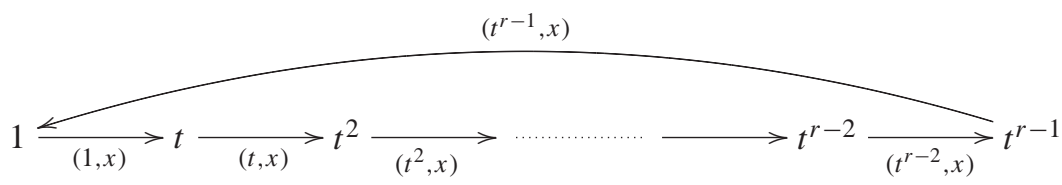

A section $\sigma_{\sim}: \mathrm{C}_{r} \rightarrow \mathrm{C}$ of $\varphi$ is given by $t^{i} \mapsto x^{i}, i=0, \ldots, r-1$, and this defines $h_{0}: \mathrm{C}_{r} \rightarrow \widetilde{F}_{1}$ by

$$
h_{0}\left(t^{i}\right)=\left(t^{i}, x^{-i}\right)
$$

It follows that for $i=0, \ldots, r-1$

$$
h_{0}\left(t^{i}\right)^{-1}\left(t^{i}, x\right) h_{0}\left(t^{i+1}\right)= \begin{cases}(1,1) & \text { if } i \neq r-1 \\ \left(1, x^{r}\right) & \text { if } i=r-1 .\end{cases}
$$

So we take a new generator $x_{2}$ for $F_{2}$ with $\delta_{2} x_{2}=x^{r}$ and set

$$
h_{1}\left(t^{i}, x\right)= \begin{cases}(1,1) & \text { if } i \neq r-1 \\ \left(1, x_{2}\right) & \text { if } i=r-1\end{cases}
$$

Then for all $i=0, \ldots, r-1$ we have

$$
\widetilde{\delta}_{2} h_{1}\left(t^{i}, x\right)=h_{0}\left(t^{i}\right)^{-1}\left(t^{i}, x\right) h_{0}\left(t^{i+1}\right) .
$$

We use next that

$$
\begin{aligned}
h_{1}\left(t^{i}, x^{r}\right) & =h_{1}\left(\left(t^{i}, x\right)\left(t^{i+1}, x\right) \ldots\left(t^{i+r-1}, x\right)\right) \\
& =\left(1, x_{2}\right) .
\end{aligned}
$$

Let $N(i)=1+t+\cdots+t^{i-1}$. Then

$$
\begin{aligned}
-h_{1} \widetilde{\delta}_{2}\left(t^{i}, x_{2}\right)+\left(t^{i}, x_{2}\right) \cdot x^{-i} & =\left(1,-x_{2}\right)+\left(1, x_{2} \cdot t^{-i}\right) \\
& =\left(1, x_{2} \cdot\left(t^{r-i}-1\right)\right) \\
& = \begin{cases}0 & \text { if } i=0, \\
N(r-i)(t-1) & \text { if } i=1, \ldots, r-1 .\end{cases}
\end{aligned}
$$

Hence we can take a new generator $x_{3}$ for $F_{3}$ with $\delta_{3} x_{3}=x_{2} \cdot(t-1)$, and define

$$
h_{2}\left(t^{i}, x_{2}\right)= \begin{cases}(1,0) & \text { if } i=0 \\ \left(1, x_{3} \cdot N(r-i)\right) & \text { if } 0<i \leqslant r-1\end{cases}
$$


Now we find that

$$
\begin{aligned}
-h_{2} \tilde{\delta}_{2}\left(t^{i}, x_{3}\right)+\left(1, x_{3} \cdot t^{-i}\right)= & -h_{2}\left(\left(t^{i-1}, x_{2}\right) \cdot t+\left(t^{i}, x_{2}\right)\right)+\left(1, x_{3} \cdot t^{-i}\right) \\
= & \begin{cases}-h_{2}\left(t^{r-1}, x_{2}\right)+\left(1, x_{3}\right) & \text { if } i=0, \\
0+h_{2}\left(t, x_{2}\right)+\left(1, x_{3} \cdot t^{r-1}\right) & \text { if } i=1, \\
\left(1, x_{3}\left(-N(r-i+1)+N(r-i)+t^{r-i}\right)\right) & \text { if } i= \\
& = \begin{cases}\left(1, x_{3} \cdot N(r)\right) & \text { if } i=1, \ldots, \\
0 & \text { otherwise. }\end{cases} \end{cases}
\end{aligned}
$$

Thus we take a new generator $x_{4}$ for $F_{4}$ with $\delta_{4} x_{4}=x_{3} \cdot N(r)$ and

$$
h_{3}\left(t^{i}, x_{3}\right)= \begin{cases}\left(1, x_{4}\right) & \text { if } i=1, \\ (1,0) & \text { otherwise }\end{cases}
$$

Then

$$
\begin{aligned}
-h_{3} \tilde{\delta}_{4}\left(t^{i}, x_{4}\right)+\left(1, x_{4} \cdot t^{-i}\right) & =-h_{3}\left(t^{i}, x_{3} \cdot N(r)\right)+\left(1, x_{4} \cdot t^{-i}\right) \\
& =-h_{3}\left(1, x_{3} \cdot N(r) \cdot t^{-i}\right)+\left(1, x_{4} \cdot t^{-i}\right) \\
& =\left(1, x_{4} \cdot\left(t^{r-i}-1\right)\right) .
\end{aligned}
$$

Thus we are now in a situation of period 2, and we have Theorem 11.3.

Theorem 11.3. A free crossed resolution $F_{*}$ of $\mathrm{C}_{r}$ may be taken to have single free generators $x_{n}$ in dimension $n \geqslant 1$ with $\varphi\left(x_{1}\right)=t$, and

$$
\delta_{n}\left(x_{n}\right)= \begin{cases}x_{1}^{r} & \text { if } n=2, \\ x_{n-1} \cdot(t-1) & \text { if } n>1, n \text { odd }, \\ x_{n-1} \cdot N(r) & \text { if } n>2, n \text { even } .\end{cases}
$$

A contracting homotopy $h_{n}$ on $\widetilde{F}_{*}$ is given by (33) for $n=0$, by (34) for $n=1$, and for $n>1$ by

$$
h_{n}\left(t^{i}, x_{n}\right)= \begin{cases}(1,0) & \text { if }\left\{\begin{array}{l}
i=0, n \text { even }, \\
i \neq 1, n \text { odd },
\end{array}\right. \\
\left(1, x_{n+1}\right) & \text { if } i=1, n \text { odd }, \\
\left(1, x_{n+1} . N(r-i)\right) & \text { otherwise. }\end{cases}
$$

\section{References}

1. Y. G. Baik, J. Harlander and S. J. Pride, 'The geometry of group extensions', J. Group Theory 1 (1998) 395-416. 30, 31

2. A. L. Blakers, 'Some relations between homology and homotopy groups', Ann. of Math. 49 (1948) 428-461. 47

3. R. Brown, 'Fibrations of groupoids', J. Algebra 15 (1970) 103-132. 49, 49 
4. R. Brown, Topology: a geometric account of general topology, homotopy types and the fundamental groupoid (Ellis Horwood, Chichester, 1988). 31, 44, 44, 46, 46, 49 , $49,50,50,50$

5. R. Brown, 'Higher order symmetry of graphs', Bull. Irish Math. Soc. 32 (1994) 46-59. 43

6. R. Brown, 'Homotopy theory, and change of base for groupoids and multiple groupoids', Applied Categorical Structures 4 (1996) 175-193. 47

7. R. Brown, 'Groupoids and crossed objects in algebraic topology', Homotopy, Homology and Applications 1 (1999) 1-78. 33

8. R. Brown, 'Higher dimensional group theory', 1997. http://www.bangor.ac.uk/ mas010/hdaweb2.html 32

9. R. Brown, P. R. Heath and H. Kamps, 'Coverings of groupoids and Mayer-Vietoris type sequences', Categorical topology, Proc. Conf. Toledo, Ohio, 1983 (Heldermann Verlag, Berlin, 1984) 147-162. 50

10. R. Brown and P. J. Higgins, 'On the connection between the second relative homotopy groups of some related spaces', Proc. London Math. Soc. (3) 36 (1978) 193-212. 47, 47

11. R. Brown and P. J.Higgins, 'On the algebra of cubes', J. Pure Appl. Algebra 21 (1981) 233-260. 46, 48

12. R. Brown and P. J. Higgins, 'Colimit theorems for relative homotopy groups', J. Pure Appl. Algebra 22 (1981) 11-41. 31, 47

13. R. Brown, and P. J. Higgins, 'Crossed complexes and non-abelian extensions', Proc. International Conference on Category Theory, Gummersbach, 1981, Lecture Notes in Math. 962 (Springer-Verlag, Berlin/New York, 1982) 39-50. 31, 51, 56

14. R. Brown and P. J. Higgins, 'Tensor products and homotopies for $\omega$-groupoids and crossed complexes', J. Pure Appl. Algebra 47 (1987) 1-33. 31, 53, 53, 53, 54

15. R. Brown and P. J. Higgins, 'Crossed complexes and chain complexes with operators', Math. Proc. Camb. Phil. Soc. 107 (1990) 33-57. 30, 36, 36

16. R. Brown and P. J. Higgins, 'The classifying space of a crossed complex', Math. Proc. Cambridge Phil. Soc. 110 (1991) 95-120. 31, 49, 52, 54

17. R. Brown and J. Huebschmann, 'Identities among relations', Low dimensional topology, London Math. Soc. Lecture Notes 46 (ed. R. Brown and T. L. Thickstun, Cambridge University Press, Cambridge, 1982) 153-202. 28, 30, 31, 33, 40, 40, 46, 46, 47

18. R. Brown and T. Porter, 'On the Schreier theory of non-abelian extensions: generalisations and computations', Proc. Roy. Irish Acad. 96A (1996) 213-227. 31, 31, 31,55

19. R. Brown and C. D Wensley, 'On finite induced crossed modules, and the homotopy 2-type of mapping cones', Theory Appl. Categ. 1 (1995) 54-71. 31, 57

20. R. Brown and C. D. WensLey., 'Computing crossed modules induced by an inclusion of a normal subgroup, with applications to homotopy 2-types', Theory Appl. Categ. 2 (1996) 3-16. 31

21. J. F. CARLSON, 'The cohomology of 2-groups mod 2'.

http://www.math.uga.edu/ jfc/groups/cohomology.html 30 
22. R. H. Crowell, 'The derived module of a homomorphism', Adv. Math. 6 (1971) 210-238. 36, 36

23. R. H. Fox, 'Free differential calculus I. Derivations in the free group ring', Ann. of Math. 57 (1953) 547-560. 30, 36

24. J. R. J. Groves, 'An algorithm for computing homology groups', J. Pure Appl. Algebra 194 (1997) 331-361. 30

25. K. W. Gruenberg, 'Resolution by relations', J. London Math. Soc. 35 (1960) 481494. 30

26. AnNe HeYwORTH, 'Applications of rewriting systems and Gröbner bases to computing Kan extensions and identities among relations', Ph.D. thesis, University of Wales, 1998. http://www.soton.ac.uk/abs/math.CT/9812097 29, 43

27. Anne Heyworth and Birgit Reinert, 'Applications of Gröbner bases to group rings and identities among relations', University of Wales Bangor Maths Preprint 99.09. http://www.bangor.ac.uk/ma/research/preprints/99/99_09.html/ 29, 36, 43, 55

28. Anne Heyworth and C. D. Wensley, 'Logged Knuth-Bendix procedures and identities among relations', University of Wales Bangor Maths Preprint 99.07. http://www.bangor.ac.uk/ma/research/preprints/99/99_07.html/ 29, 35, 43, 55

29. P. J. Higgins, 'Presentations of groupoids, with applications to groups', Proc. Camb. Phil. Soc. 60 (1964) 7-20. 31, 31, 34, 36

30. P. J. Higgins, Categories and groupoids (Van Nostrand, London, 1971). 49

31. C. Hog-Angeloni, W. Metzler and A. J. Sieradski (Eds), Two-dimensional homotopy and combinatorial group theory, London Math. Soc. Lecture Note Series 197 (Cambridge University Press, Cambridge, 1993). 30, 33, 46

32. J. HowIE, 'Pullback functors and crossed complexes', Cahiers Topologie Géom. Différentielle Catég. 20 (1979) 281-295. 32, 49, 51

33. S. T. Hu, Homotopy theory (Academic Press, New York, 1959). 50

34. J. Huebschmann, 'Crossed $n$-fold extensions and cohomology', Comment. Math. Helv. 55 (1980) 302-314. 55, 56

35. L. Johansson, L. LAmbe and E. Sköldberg, 'Normal forms and iterative methods for constructing resolutions', Preprint, Stockholm, 1998. 30

36. S. J. PRIDE, 'Identities among relations', Proc. Workshop on Group Theory from a Geometrical Viewpoint, International Centre of Theoretical Physics, Trieste, 1990 (ed. E. Ghys, A. Haefliger and A. Verjodsky, World Scientific, Singapore, 1991) 687-716. 30

37. M. SCHÖNERT et al., 'GAP: groups, algorithms, and programming', 4th edn (Lehrstuhl D für Mathematik, Rheinisch Westfälische Technische Hochschule, Aachen, Germany, 1997).

38. J. Sмітн, 'Equivariant Moore spaces', Algebraic and geometric topology, Proceedings Rutgers 1983, Springer Lecture Notes in Math. 1126 (ed. A. Ranicki, N. Levitt and F. Quinn, Springer-Verlag, Berlin/New York, 1985) 238-270. 30

39. A. P. Tonks, 'Theory and applications of crossed complexes', $\mathrm{PhD}$ thesis, University of Wales, Bangor, 1993. http://www.bangor.ac.uk/ma/research/tonks 31, 55, 56

40. J. H. C. Whitehead, 'Combinatorial homotopy II', Bull. Amer. Math. Soc. 55 (1949) 453-496. 30, 30, 31, 33, 36, 36, 46, 47, 47, 47 
Ronald Brown r.brownebangor.ac.uk http://www.bangor.ac.uk/ mas010 School of Mathematics

University of Wales

Dean Street, Bangor

Gwynedd LL57 1UT, U.K.

Abdul Razak Salleh aras@pkrisc.cc.ukm.my

Department of Mathematics, Faculty of Mathematical Sciences

Universiti Kebangsaan Malaysia

43600 Bangi

Selangor D.E.

Malaysia 